\title{
Peritumoral implantation of hydrogel-containing nanoparticles and losartan for enhanced nanoparticle penetration and antitumor effect
}

This article was published in the following Dove Press journal: International Journal of Nanomedicine

\author{
Haijun Shen' \\ Qianqian Gao' \\ Qi Yel \\ Shiyun Yang' \\ Yuqian $\mathrm{Wu}^{\prime}$ \\ Qin Huang' \\ Xiaona Wang ${ }^{2}$ \\ Zhenhua Sun ${ }^{3}$ \\ 'Department of Preventive Medicine \\ and Public Health Laboratory Science, \\ School of Medicine, Jiangsu University, \\ Zhenjiang, Jiangsu Province, China; \\ ${ }^{2}$ Department of Internal Medicine of \\ Jiangsu University Hospital Workers, \\ The Affiliated Hospital of Jiangsu \\ University, Zhenjiang, Jiangsu Province, \\ China; ${ }^{3}$ Department of Thyroid and \\ Breast Surgery, The Affiliated Hospital \\ of Jiangsu University, Zhenjiang, \\ Jiangsu Province, China
}

Correspondence: Haijun Shen School of Medicine, Jiangsu University, 301 Xuefu Road, Zhenjiang 212013, Jiangsu Province, China

Emailshenhj@ujs.edu.cn

Zhenhua Sun

The Affiliated Hospital of Jiangsu University, 438 Jiefang Road, Zhenjiang 2I200I, Jiangsu Province, China Emailbjxh100@I63.com
Background and purpose: Nanoparticle-loaded hydrogels - localized drug delivery devices containing a combination of therapeutic nanoparticles and implantable hydrogel - have been recipients of increased focus and interest for cancer treatment. However, it is difficult for the released nanoparticles to penetrate deeply into tumors because of the dense collagen network in the tumor extracellular matrix, which greatly limits their antitumor effect. We hypothesized that the implantation of a hydrogel loaded with both nanoparticles and losartan (Los) might enhance penetration because Los has been proven to effectively reduce collagen levels in various tumors. Herein, we developed a nanoparticle/Los-loaded hydrogel system and evaluated the intratumoral distribution and anticancer effect after peritumoral implantation of nanoparticles.

Methods: Fluorescent polystyrene nanoparticles (FPNPs, size $\sim 100 \mathrm{~nm}$ ) and Los were simultaneously encapsulated in a polyethylene glycol (PEG) hydrogel to form the FPNP/ Los-loaded hydrogel. After peritumoral implantation in 4T1 tumor-bearing mice for 2 weeks, intratumoral distributions of FPNPs and collagen level were determined. Based on the results, liposomal doxorubicin (Doxil, $\sim 100 \mathrm{~nm}$ ) was subsequently substituted for FPNPs in the hydrogel. The cellular uptake and cytotoxicity of the Doxil/Los-loaded hydrogel were studied, and the in vivo antitumor efficacy after peritumoral implantation was evaluated.

Results: Compared with a standard FPNP-loaded hydrogel, the FPNP/Los-loaded hydrogel resulted in enhanced penetration and reduced collagen levels after implantation. Thereafter, the potential of a Doxil/Los-loaded hydrogel for cancer treatment was studied. Doxorubicin was released from the hydrogel and induced effective cytotoxicity against 4T1 cells. The Doxil/ Los-loaded hydrogel showed synergistic antitumor effects in 4T1 tumor-bearing mice and was more effective at tumor inhibition than the Doxil-loaded hydrogel.

Conclusion: This study provides a proof of principle that the implantation of nanoparticles/ Los-loaded hydrogel can increase the intratumoral distribution and antitumor efficacy of nanoparticles, owing to collagen depletion by Los. Future studies may build on this strategy for enhanced tumor penetration of nanoparticles.

Keywords: localized drug delivery, tumor penetration, tumor extracellular matrix, collagen, matrix metalloproteinase, breast cancer

\section{Introduction}

Cancer is a major public health problem and remains a leading cause of death worldwide. Various cancer treatment strategies have been developed. In the past decades, nanocarrierbased drug delivery systems have attracted increasing attention. ${ }^{1-3}$ The treatment of tumors can be improved by using nanoparticulate formulations to deliver antitumor agents to cancer tissues, via passive or active targeting. However, the intravenously 
delivered nanoparticles still display some deficiencies, such as rapid clearance from the bloodstream with subsequent accumulation in non-target organs, overreliance on the enhanced permeability and retention effect to transport nanoparticles into the tumor, and, consequently, achievement of only a modest increase in tumor accumulation. ${ }^{4-6}$ To address some of these problems, localized drug delivery by direct administration or implantation of antitumor agents to tumor sites is a promising strategy. Such localized systems can ensure high drug concentrations in cancerous tissues for extended periods of time whereas simultaneously maintaining low systemic drug exposure, which may translate into minimized systemic side effects, reduced doses, and less frequent administration. ${ }^{7-10}$ However, free drugs or nanoparticle drug depots (eg, emulsions, liposomes, and micelles) directly injected into tumor sites easily migrate away from the target location. ${ }^{11}$ In this context, biocompatible polymeric hydrogels, which can improve patient compliance and increase drug retention at tumor sites, are commonly used for localized drug delivery.

More recently, nanoparticle drug depots have been encapsulated in hydrogels to develop novel nanoparticle-encapsulated hydrogel systems. ${ }^{11-13}$ Such combination drug delivery systems are able to effectively integrate the advantages of nanoparticles and hydrogels. For example, after encapsulation in the hydrogel, nanoparticles cannot easily migrate away and thus show sustained release in the implant location. Moreover, nanoparticle drug depots are loaded into the hydrogel by a gentle physical embedding process. Therefore, the released nanoparticles still exhibit their inherent features, such as specific targeting of tumor cells, enhanced cellular uptake, and intracellular distribution. Yang et al developed a localized drug delivery device by combining an active-targeting micellar system and implantable polymeric nanofibers. In contrast to the systematic administration of therapeutics via repeated intravenous injections of micelles, this implantable device enabled efficient and safe cancer therapy with reduced drug dose and administration frequency. ${ }^{11}$ Overall, implantable nanoparticles-in-hydrogel systems show promise for the treatment of local and regional malignancies.

Therapeutic nanoparticles, regardless of whether they are delivered by intravenous administration or localized implantation, should be transported through the interstitial compartment to reach cancer cells. Although the localized delivery of nanoparticles by hydrogel systems exhibits unique advantages, as described earlier, it still cannot overcome the poor penetration of nanoparticles in tumors. The tumor extracellular matrix (ECM) has been acknowledged as one major barrier to the intratumoral penetration of nanoparticles, especially the dense network of collagen. ${ }^{14,15}$ Collagen constitutes up to $90 \%$ of ECM and is widespread in breast cancers and pancreatic cancers. ${ }^{16}$ As tumors progress, the ECM becomes stiffer and thicker, owing to increased collagen synthesis and deposition. ${ }^{17}$ This increased ECM deposition directly contributes to tumor growth, although it greatly hinders the penetration of nanoparticles into the tumor. ${ }^{16,18,19}$ As the main component of tumor ECM is collagen, falling levels of collagen in tumors may increase the penetration of nanoparticles and thus lead to a better anticancer effect. So far, several strategies have been developed to destroy the collagen lattice and enhance the penetration of therapeutic nanoparticles, for instance, using matrix modifiers (eg, collagenase $)^{20,21}$ and collagen production inhibitors such as TGF- $\beta$ inhibitors or losartan (Los). ${ }^{16,22}$

As an angiotensin inhibitor, Los has been extensively used in clinical settings to control hypertension in patients. More recently, Los has exhibited remarkable effects in reducing collagen levels in solid tumors via an angiotensin II type I receptor, which mediates the downregulation of tumor TGF- $\beta 1.16,17,22,23$ TGF- $\beta$ is a key promoter of cancer-associated fibroblast (CAF) activity. ${ }^{23}$ Because the collagen in tumors is mostly produced by CAFs, the downregulation of TGF- $\beta$ results in a reduced collagen level in tumors. ${ }^{22}$ Moreover, it has been widely shown that Los can improve the penetration of nanoparticles in various tumor types, including breast cancer, pancreatic cancer, lung cancer, fibrosarcoma, and melanoma. ${ }^{16,17,19,24,25}$

Overall, although nanoparticles-in-hydrogel systems can be used to ensure high nanoparticulate drug exposure and sustained drug release in the tumor area, the tumor ECM still hampers the intratumoral penetration and therapeutic effectiveness of the released nanoparticles. In this study, Los and nanoparticles were simultaneously encapsulated in a polyethylene glycol (PEG) hydrogel crosslinked by matrix metalloproteinase (MMP)-cleavable peptides. This nanoparticle/Los-loaded hydrogel was expected to be degraded by MMP and release its cargo after peritumoral implantation. We hoped that the collagen network would be depleted by the released Los, resulting in enhanced penetration of nanoparticles into the tumor, as depicted in Figure 1. To demonstrate the validity of our approach, first, fluorescent polystyrene nanoparticles (FPNPs) were used as a model for the $100 \mathrm{~nm}$ particles because of their low-cost and detectable fluorescence. The intratumoral penetration of FPNPs was evaluated after the implantation of an FPNP/ Los-loaded hydrogel into 4T1 breast tumor-bearing mice. Collagen levels were determined by Masson's trichrome staining assay. After this validation, the applicability of 


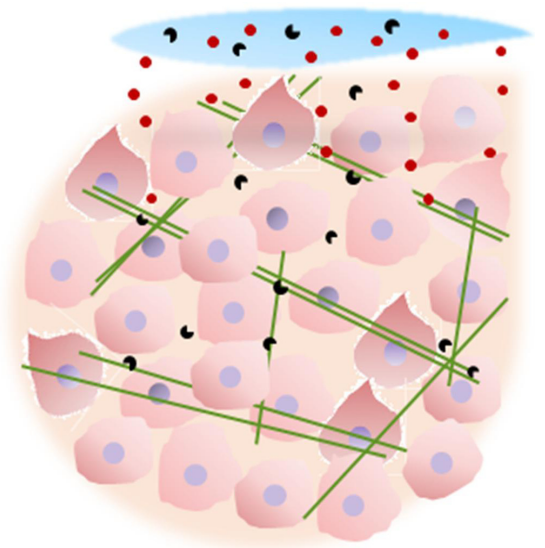

I) Cargos were released from hydrogel

II) Delivered into tumor

III) Nanoparticle penetration was hindered

IV) Collagen was decreased by Los

V) Enhanced nanoparticle penetration

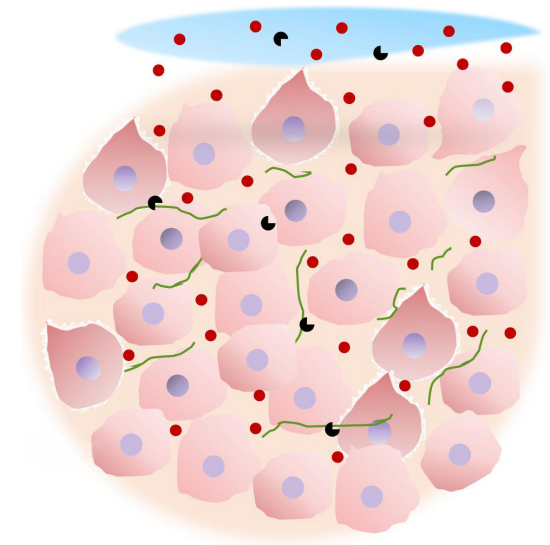

Figure I Schematic illustration of the enhanced intratumoral penetration of nanoparticles after peritumoral implantation of nanoparticle/Los-loaded hydrogel. Notes: In this work, FPNPs and Doxil $(\sim 100 \mathrm{~nm})$ were employed as model nanoparticles. After peritumoral implantation of the nanoparticle/Los-loaded hydrogel, nanoparticles and Los could be released from the hydrogel and delivered into the tumor through blood vessels and lymphatic capillaries. The dense collagen networks in tumors greatly hinder the deep penetration of nanoparticles. Los effectively reduces collagen levels, thereby enhancing the intratumoral distribution of nanoparticles. Abbreviations: Doxil, liposomal doxorubicin; FPNPs, fluorescent polystyrene nanoparticles; Los, losartan.

this nanoparticle/Los-loaded hydrogel system for cancer treatment was further studied. Doxil - a liposomal doxorubicin (DOX) with an average size $\sim 100 \mathrm{~nm}$ - was encapsulated in hydrogels to develop a Doxil/Los-loaded hydrogel system. The cellular uptake of DOX, its in vitro cytotoxicity against $4 \mathrm{~T} 1$ cells, and the in vivo antitumor effect after peritumoral implantation were studied. The 4T1 tumor model was used in this study because of its high expression of collagen I, which constitutes the majority of collagen networks in tumors. Moreover, the overexpression of MMP in 4T1 tumors was expected to favor hydrogel degradation and cargo release.

\section{Materials and methods Materials}

The MMP-cleavable peptide GCRDGPQGIWGQDRCG (HSMMP-SH) was synthesized by GenScript Co. Ltd. (Nanjing, China). Four-arm polyethylene glycol maleimide (4-arm PEGMal, Mw $20 \mathrm{kDa}$ ) was supplied by Jenkem Technology Co. Ltd. (Beijing, China). Los was purchased from MedChemExpress (New Jersey, NJ, USA). Fluorescent polystyrene sulfatemodified latex beads (L9902-1 ML) and collagenase were obtained from Sigma-Aldrich Co. (St Louis, MO, USA). Doxil was purchased from Avanti Polar Lipids (Alabaster, AL, USA). LysoTracker Green, 4',6-diamidino-2-phenylindole (DAPI), LIVE/DEAD ${ }^{\text {TM }}$ Cell Imaging Kit, Roswell Park Memorial Institute (RPMI) 1640 medium, FBS, trypsin, and penicillinstreptomycin were all purchased from Thermo Fisher Scientific (Waltham, MA, USA). The Cell Counting Kit-8 (CCK-8) assay was obtained from Dojindo (Kumamoto, Japan). All other reagents, which were of at least reagent grade, were purchased from commercial sources and used as received.

\section{Preparation of FPNP/Los-loaded hydrogel}

In total, 4-arm PEG-Mal was dissolved in PBS ( $\mathrm{pH} 7.4$ ) to obtain a $0.1 \mathrm{mg} / \mu \mathrm{L}$ solution. HS-MMP-SH was dissolved in triethylamine buffer $(\mathrm{pH} \mathrm{8.0)}$ to a final concentration of $0.1 \mathrm{mg} / \mu \mathrm{L}$. A $0.8 \mathrm{mg} / \mu \mathrm{L}$ Los solution was prepared in deionized water. In a typical gel preparation, $100 \mu \mathrm{L}$ 4-arm PEG-Mal solution was mixed well with $20 \mu \mathrm{L}$ FPNPs and $10 \mu \mathrm{L}$ Los solution. Afterward, $20 \mu \mathrm{L}$ HS-MMP-SH solution was added under vortexing. Gelation occurred within a few minutes. The crosslinking reaction was continued for $\sim 30$ minutes at $37^{\circ} \mathrm{C}$. The PEG hydrogel was then formed, and both FPNPs and Los were encapsulated in the hydrogel (abbreviated as FPNP/Los-loaded hydrogel).

\section{Characterization of hydrogel}

The resulting hydrogel was lyophilized and placed on a double-sided carbon tape and sputter-coated with gold-palladium in an argon atmosphere. The morphology of the FPNP/Los-loaded hydrogel was observed by field emission scanning electron microscopy (SEM, S-4800; Hitachi Ltd., Tokyo, Japan).

The MMP-sensitive property of the hydrogel was characterized by studying the in vitro release of FPNPs from the hydrogel. Briefly, FPNP/Los-loaded hydrogels were suspended in $1 \mathrm{~mL}$ of 
$10 \mathrm{mM}$ PBS (pH 7.4), with collagenase $(0.1 \mathrm{U} / \mathrm{mL})$ or without collagenase, in Eppendorf tubes that were gently rotated at $37^{\circ} \mathrm{C}$. At predetermined time points, supernatants were collected and tubes were replenished with the same volume of fresh-release solution. The fluorescence of FPNPs released from hydrogels was determined using a multimode microplate reader (Synergy HT; BioTek, Winooski, VT, USA) with $\lambda_{\mathrm{ex}} \sim 538 \mathrm{~nm}$ and $\lambda_{\mathrm{em}} \sim 584 \mathrm{~nm}$. The cumulative release of FPNPs was calculated with the help of a standard curve (data not shown).

\section{Cell culture and animals}

The 4T1 cells (murine breast cancer cell line) were from the Chinese Academy of Sciences Cell Bank (Shanghai, China). Cells were cultured in RPMI 1640 medium supplemented with $10 \%$ (v/v) FBS, 1\% (v/v) $100 \mathrm{U} / \mathrm{mL}$ streptomycin, and $1 \%(\mathrm{v} / \mathrm{v}) 100 \mathrm{U} / \mathrm{mL}$ penicillin. The cells were maintained at $37^{\circ} \mathrm{C}$ in a humidified $5 \% \mathrm{CO}_{2}$ atmosphere.

Four-week-old female BALB/c nude mice were purchased from Nanjing Biomedical Research Institute of Nanjing University (Nanjing, China) and kept under specific pathogenfree conditions. All animal experiments were conducted in accordance with the guidelines specified for laboratory animals by the Ethics Committee of Jiangsu University (Zhenjiang, China). The Animal Care and Use Committee of Jiangsu University approved the use of animals in this study.

\section{Intratumoral distribution of nanoparticles}

The 4T1 subcutaneous tumor model was established as in previous studies. ${ }^{17}$ In short, $10^{6} 4 \mathrm{~T} 1$ cells were subcutaneously injected into the right axilla of female Balb/c nude mice weighing $\sim 18-20 \mathrm{~g}$. When the tumors reached approximately $0.5 \times 0.5 \mathrm{~cm}$ in diameter, the mice were randomly assigned into either the FPNP-loaded hydrogel group or the FPNP/ Los-loaded hydrogel group $(n=3)$. Mice in the FPNP-loaded hydrogel group were implanted with hydrogels loaded with $20 \mu \mathrm{L}$ FPNPs, whereas those in the FPNP/Los-loaded hydrogel group were implanted with hydrogels loaded with $20 \mu \mathrm{L}$ FPNPs and $8 \mathrm{mg}$ Los.

For hydrogel implantations (Figure 2), the mice were anesthetized by an intraperitoneal injection of trichloroacetaldehyde hydrate solution ( $2.5 \%$ in saline, $0.2 \mathrm{~mL}$ per mouse) prior to surgery. After disinfecting, a skin incision of $\sim 1 \mathrm{~cm}$ was made alongside the tumor mass. At this site, a subcutaneous pocket was created along the tumor mass, and the hydrogel was subsequently inserted and coated on the tumor. Finally, the skin wound was closed with surgical sutures.

After 2 weeks of treatment, all mice were sacrificed and tumors were collected. Parts of the tumors were fixed in formalin solution for Masson's trichrome staining assay. The remaining tumor portions were frozen, embedded in optimal cutting temperature (OCT) compound, and cryosectioned. We then mounted $10 \mu \mathrm{m}$ sections on microscopic slides; the red fluorescence generated by the FPNPs was visualized and qualitative nanoparticle intratumoral distribution was analyzed using the ImageJ software. On average, nine images were analyzed for each group.

\section{Masson's trichrome staining assay}

The tumors were fixed in formalin for at least 24 hours, embedded in paraffin blocks, and then sectioned. Masson's
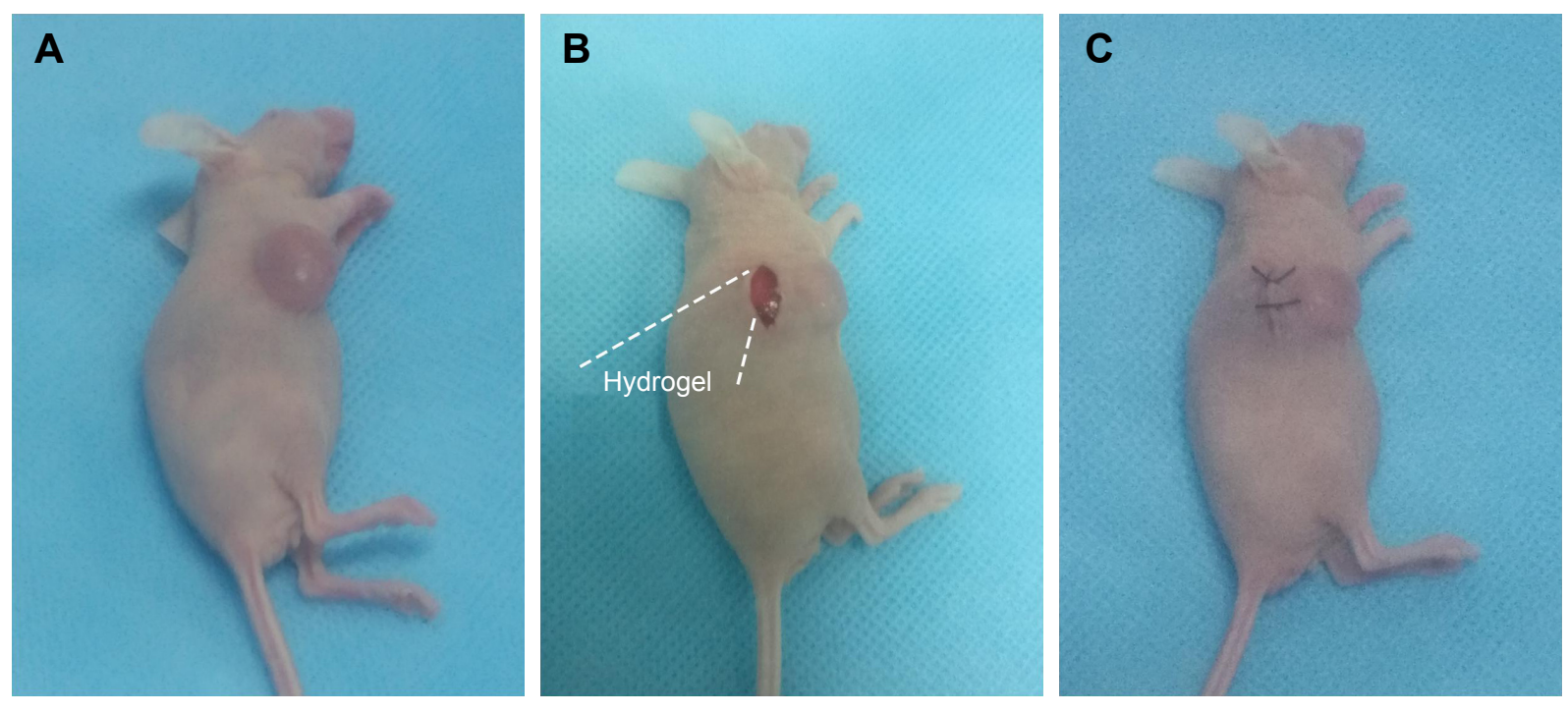

Figure 2 Process of peritumoral implantation of hydrogel.

Notes: (A) A mouse bearing a 4TI cell-xenografted tumor is anesthetized; (B) a skin incision is made beside the tumor mass and the hydrogel is implanted; and (C) the incision is sutured. 
trichrome staining was then undertaken to localize the collagen distribution in the tumors. In this assay, blue staining indicates the presence of collagen fibers in the tumor. The quantification of Masson trichrome-stained collagen expression was undertaken using ImageJ. Data were averaged from six images per group.

\section{Preparation and characterization of Doxil/Los-loaded hydrogel}

To evaluate the potential of the nanoparticle/Los-loaded hydrogel for cancer treatment, Doxil (Avanti Polar Lipids, Inc.) - instead of FPNPs - was loaded in a PEG hydrogel via the same protocol. The morphology of the Doxil/Los-loaded hydrogel was observed by SEM after lyophilization. Interconnected pore diameters were manually measured using ImageJ from SEM images taken at $200 \times$ magnification; the average diameter was obtained by averaging random 50 pores from two images. ${ }^{26}$

To study Doxil release from the hydrogel, two pieces of $150 \mu \mathrm{L}$ Doxil/Los-loaded hydrogels were immersed in $2 \mathrm{~mL}$ PBS (10 mM, pH 7.4), with collagenase $(0.1 \mathrm{U} / \mathrm{mL})$ or without collagenase, in $5 \mathrm{~mL}$ centrifuge tubes, respectively. After gentle shaking at $37^{\circ} \mathrm{C}$ for 12 hours, $1 \mathrm{~mL}$ of liquid was collected from each tube. The mean diameter and zeta potential of the released Doxil were measured using a Malvern Zetasizer Nano ZS90 (Malvern Instruments, Malvern, UK). Measurements were repeated three times. Further, the release medium was dripped onto a copper grid coated with amorphous carbon and then observed under transmission electron microscopy (TEM; Tecnai 12, Philips, the Netherlands) after being stained by phosphotungstic acid (1 wt.\%). Unincorporated Doxil was used as a control.

\section{Cellular uptake of DOX from hydrogel}

The 4T1 cells were seeded into $15 \mathrm{~mm}$ glass-bottomed culture dishes with densities of $1.5 \times 10^{5}$ cells/dish. The precursor solution for the hydrogel was spread on a coverslip to allow for gelation. After the cells were attached and well dispersed, coverslips with Doxil-loaded hydrogel and Doxil/Los-loaded hydrogel were immersed in the culture medium and fixed between two rings. ${ }^{11}$ The final DOX concentration in the medium was equivalent to $5 \mu \mathrm{g} / \mathrm{mL}$, and the Los concentration was $8 \mu \mathrm{g} / \mathrm{mL}$. After incubation for 4 hours, the hydrogel and medium were discarded. Then, LysoTracker Green was added to a final concentration of $100 \mathrm{nM}$ and incubated for 30 minutes. After treatment, cells were washed twice with cold PBS and fixed with 4\% (w/v) paraformaldehyde. The nuclei of cells were stained with DAPI. The cellular uptake and intracellular localization of DOX released from the hydrogel were then observed using DeltaVision Elite Cell Imaging System (GE Healthcare, Issaquah, WA, USA).

In view of the intrinsic fluorescence of DOX, the cellular uptake of DOX was further quantitatively analyzed by flow cytometry. The $4 \mathrm{~T} 1$ cells were seeded in a 12-well plate at a density of $1.0 \times 10^{5}$ cells/well and cultured overnight. The Doxil-loaded hydrogels and Doxil/Los-loaded hydrogels were immersed in $1 \mathrm{~mL}$ culture medium in another 12-well plate and maintained in the cell incubator to allow drug release. The final DOX concentration in the medium was equivalent to $5 \mu \mathrm{g} / \mathrm{mL}$, and the Los concentration was $8 \mu \mathrm{g} / \mathrm{mL}$. After 4 hours, the drug-containing medium was transferred into the 12-well plate with adherent cells for cellular uptake. After incubation for 4 hours, cells were washed with PBS, trypsinized, and centrifuged. Cells were re-suspended in PBS and analyzed using a flow cytometer (BD Biosciences, San Jose, CA, USA). Data obtained were analyzed using the FlowJo software. Cells cultured in normal medium without Doxil were used as a control.

\section{In vitro cytotoxicity assay}

For cytotoxicity evaluation of the hydrogels, a live/dead assay was initially conducted. Briefly, $2 \times 10^{4} 4 \mathrm{~T} 1$ cells were seeded in each well of a 24 -well plate and incubated for 12 hours. The blank hydrogel, Doxil-loaded hydrogel, and Doxil/Los-loaded hydrogel were separately loaded into porous polyester Transwell ${ }^{\mathrm{TM}}$ inserts (pore size $5.0 \mu \mathrm{m}$ ) and then placed in each well of the cell culture plate. These inserts were immersed in culture medium with or without $0.1 \mathrm{U} / \mathrm{mL}$ collagenase. The final DOX concentration in the medium was equivalent to $5 \mu \mathrm{g} / \mathrm{mL}$, and the Los concentration was $8 \mu \mathrm{g} / \mathrm{mL}$. After incubation for 24 hours, cells were washed thrice with PBS after medium removal and then stained using a LIVE/DEAD ${ }^{\mathrm{TM}}$ Cell Imaging Kit. The stained live 4T1 cells (green) and dead 4T1 cells (red) were viewed using a fluorescence microscope (Carl Zeiss Meditec AG, Jena, Germany).

Furthermore, the cell viability was assessed by the CCK-8 assay. Briefly, 4T1 cells were seeded in a 24 -well plate at a density of $2 \times 10^{4}$ cells/well. After the cells attached and were well spread, the blank, Doxil-loaded, and Doxil/Los-loaded hydrogels were immersed in the culture medium with or without $0.1 \mathrm{U} / \mathrm{mL}$ collagenase. The final concentrations of DOX and Los in media were 5 and $8 \mu \mathrm{g} / \mathrm{mL}$, respectively. As a control, cells were incubated with normal culture medium. After cultivation for 24 and 48 hours, the medium was removed, the CCK-8 solution in medium was added to each well, and cells were further incubated for 1 hour. Finally, $100 \mu \mathrm{L}$ medium per well from the 24-well plate 
was transferred to a 96-well plate, and the absorbance was recorded at $450 \mathrm{~nm}$ using a microplate reader (BioTek).

\section{In vivo antitumor activity}

The 4T1 tumor-bearing mice were established using the aforementioned protocol. When the tumors reached $\sim 0.5 \times 0.5 \mathrm{~cm}^{3}$ in size, mice were randomly divided into four groups $(n=5)$. Different treatments were then applied to the mice (labeled as Day 0). Thereafter, the blank, Doxil-loaded, and Doxil/ Los-loaded hydrogels were implanted subcutaneously near the tumors at a $3 \mathrm{mg} / \mathrm{kg}$ (DOX/mouse weight) equivalent DOX dosage and $400 \mathrm{mg} / \mathrm{kg}$ Los dosage. Mice without any treatment were used as the control group. Tumor volumes were recorded every other day. The tumor volume was calculated using the formula $V=(L \times W \times W) / 2$ (where $L$ and $W$ are the longest and shortest dimensions, respectively). All the mice were sacrificed on Day 18, and the tumors from each group were excised, photographed, and weighed. The tumor inhibitory rate (TIR) was calculated using the following equation:

$$
\mathrm{TIR}=\left(1-\frac{\mathrm{W}_{\text {test }}}{\mathrm{W}_{\text {control }}}\right) \times 100 \%
$$

where $\mathrm{W}_{\text {test }}$ is the mean tumor weight of the tested groups, and $\mathrm{W}_{\text {control }}$ is the mean tumor weight of the control group.

\section{Statistical analysis}

The results were presented as mean \pm SD. Statistical analysis was conducted using the Student's $t$-test. Differences between groups were considered statistically significant at $P<0.05(*)$.

\section{Results \\ Preparation and characterization of FPNP/Los-loaded hydrogel}

For local chemotherapy using nanoparticles and Los simultaneously, an implantable PEG hydrogel was employed as the carrier. Herein, PEG hydrogel was formed in situ via a thiol-maleimide reaction between the 4-arm PEG-Mal and HS-MMP-SH (crosslinker) at ambient temperature. The thiol-maleimide reaction was chosen because it is rapid and controllable. ${ }^{27}$ Throughout this in situ crosslinking reaction, the nanoparticles and Los were simultaneously encapsulated in the gel matrix. As shown in Figure 3A and B, combining solutions of 4-arm PEG-Mal, FPNPs, Los, and HS-MMP-SH resulted in a non-liquid and transparent pink hydrogel. The internal structure of the hydrogel was observed by SEM (Figure 3C). The PEG network and porous structure could be clearly observed, indicating that the developed PEG hydrogel might be an excellent vehicle for nanoparticles and Los. The pink appearance of the hydrogel visually demonstrated the encapsulation of pink FPNPs.

To characterize the MMP-sensitive property of the PEG hydrogel, the release profile of FPNPs from hydrogels was investigated for 48 hours (Figure 4A). In the absence of collagenase, the hydrogel showed a lower release rate for FPNPs, with the maximum released amounts being less than $15 \%$. However, in the presence of collagenase $(0.1 \mathrm{U} / \mathrm{mL})$, significantly faster release of FPNPs was observed. During 48-hour incubation, the release profiles of FPNPs showed an initial burst for the first 10-hour period, and $\sim 100 \%$ release was achieved. Thus, it was clear that collagenase accelerated the release of FPNPs from hydrogels. Furthermore, the degradation of MMP-sensitive hydrogels was investigated (Figure 4B).

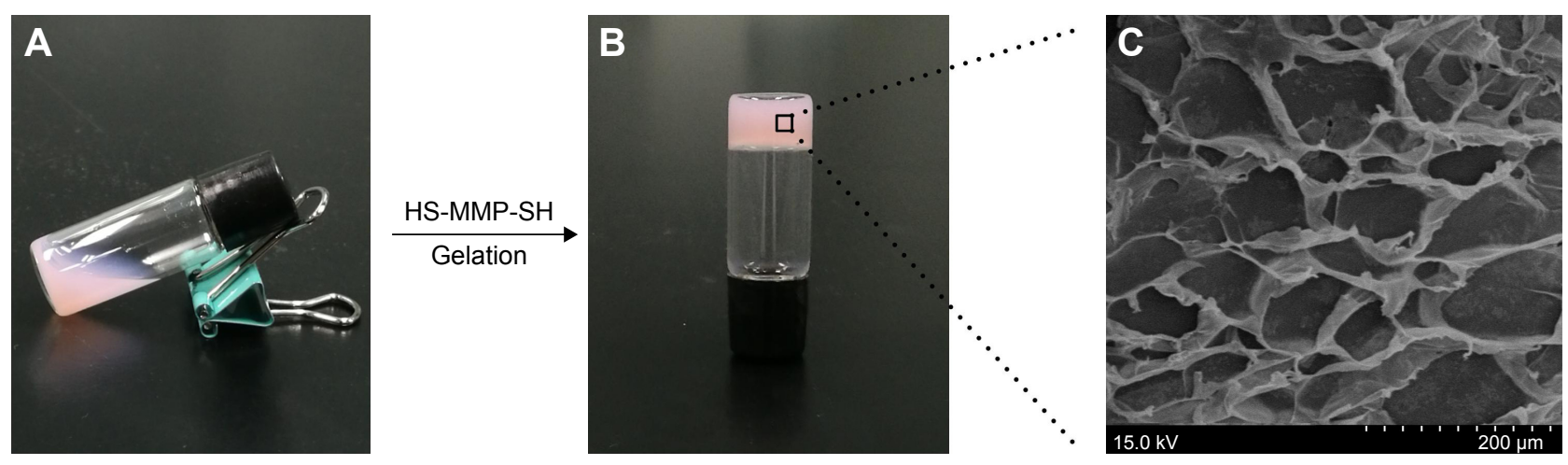

Figure 3 (A) Photograph of the solution mixture containing 4-arm PEG-Mal, FPNPs, and Los. (B) Photograph of the FPNP/Los-loaded hydrogel. (C) SEM image of the FPNP/ Los-loaded hydrogel.

Notes: After addition of HS-MMP-SH, gelation quickly occurred owing to the thiol-maleimide reaction between PEG-Mal and HS-MMP-SH, resulting in the encapsulation of FPNPs and Los. HS-MMP-SH, matrix metalloproteinases cleavable peptide.

Abbreviations: FPNPs, fluorescent polystyrene nanoparticles; Los, losartan; PEG-Mal, polyethylene glycol maleimide; SEM, scanning electron microscopy. 
A

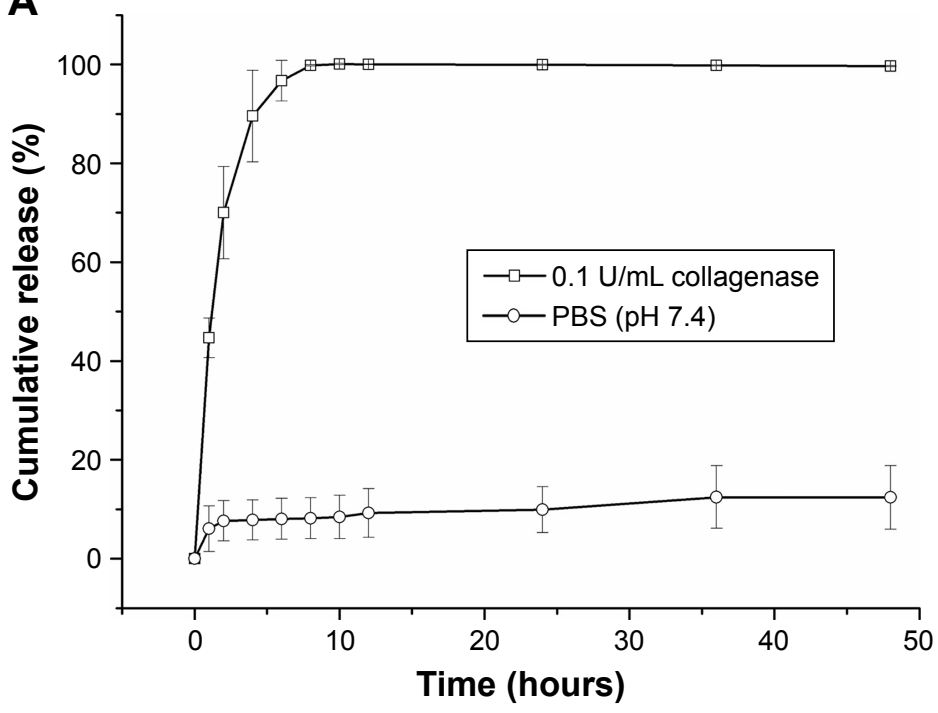

B

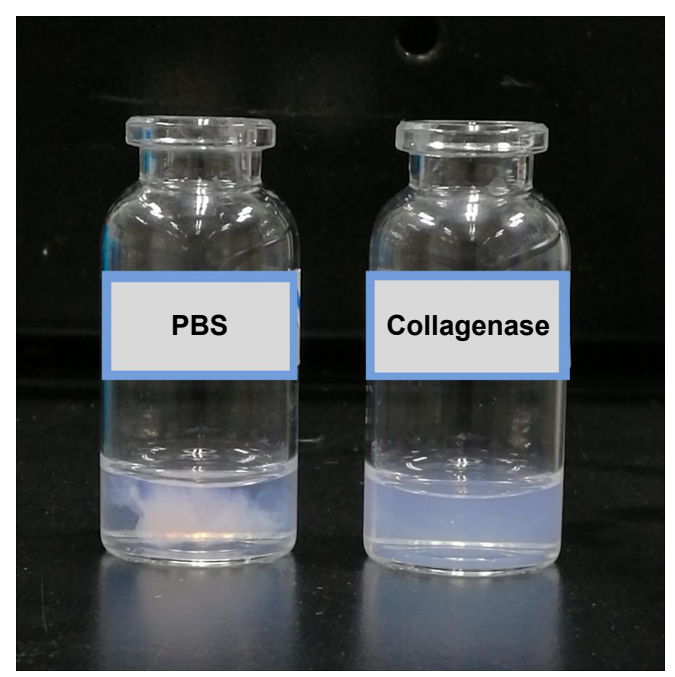

Figure 4 Characterization of MMP-sensitive property of the PEG hydrogel.

Notes: (A) Cumulative release of FPNPs from FPNP/Los-loaded hydrogel in PBS solution with or without collagenase; (B) photographs of the FPNP/Los-loaded hydrogels after I2-hour immersion in PBS solution with or without collagenase.

Abbreviations: FPNPs, fluorescent polystyrene nanoparticles; Los, losartan; MMP, matrix metalloproteinase; PEG, polyethylene glycol.

After immersion in PBS buffer (pH 7.4, no collagenase) for 12 hours, the FPNP-loaded hydrogel was still visible in the release medium, like a cluster of clouds. By contrast, the medium appeared homogeneous and transparent after immersion for 12 hours in the presence of $0.1 \mathrm{U} / \mathrm{mL}$ collagenase, indicating the complete degradation of the hydrogel. This result provided a reasonable explanation for the abovementioned difference in release profiles in the absence or presence of collagenase: FPNPs incorporated in the hydrogels were slowly released from the matrix by simple diffusion without cleavage of MMP-responsive peptides in the absence of collagenase, whereas FPNPs were released by enzymatic cleavage of MMP-responsive peptides and subsequent degradation of the hydrogel matrix in the presence of collagenase.

The faster release and degradation of hydrogel in the presence of collagenase demonstrated the MMP-sensitive property of the PEG hydrogel. MMPs, especially MMP-2 and MMP-9, are known to be involved and overexpressed in many stages of human cancers. ${ }^{28}$ They are key effectors of angiogenesis and invasion of cancer cells. Levels of MMP are high at the invasive edge of tumors as well as at the sites of angiogenesis. These conditions make enzymatic degradation by MMP a highly favorable trigger mechanism. ${ }^{29}$ Various MMP-sensitive drug delivery systems have been designed and have shown stimulus responsiveness. ${ }^{28-30}$ In this work, MMP-sensitive peptides were employed to crosslink 4-arm PEG-Mal to generate the FPNP/Los-loaded hydrogel. In a tumor microenvironment, the peptide linker would be cleaved by the upregulated extracellular MMP, allowing the liberation of the therapeutic nanoparticles and Los. Therefore, the MMP-sensitive property of the hydrogel was expected to be beneficial for its degradation in vivo and for drug delivery after implantation.

\section{Effects of Los on intratumoral distribution of nanoparticles}

To evaluate the tumor-penetration efficiency of FPNPs with or without treatment of Los, ultrathin slices were prepared and the fluorescence distribution was observed. In control tumors (implantation of FPNP-loaded hydrogels without Los), the intratumoral penetration of FPNPs was limited and most of the nanoparticles were found in the tumor margin. There was little or no nanoparticle accumulation in the center of tumors (Figure 5A and C). Conversely, after implantation of the FPNP/Los-loaded hydrogel for 2 weeks, a significant increase in the accumulation and penetration of FPNPs was evidenced by the observed intense and uniform distribution of nanoparticles in the tumor center (Figure 5B and D). The quantitative fluorescent data indicated significant improvement in intratumoral distribution in the Los-treated group as compared with the untreated control group. When compared with controls, the FPNP/Los-loaded hydrogel group showed 2.98 -fold increases in the intratumoral fluorescent area as a result of FPNP distribution (Figure 5E). These results suggest that localized and prolonged peritumoral delivery of Los was effective in enhancing the tumor distribution and penetration of the nanoparticles. 

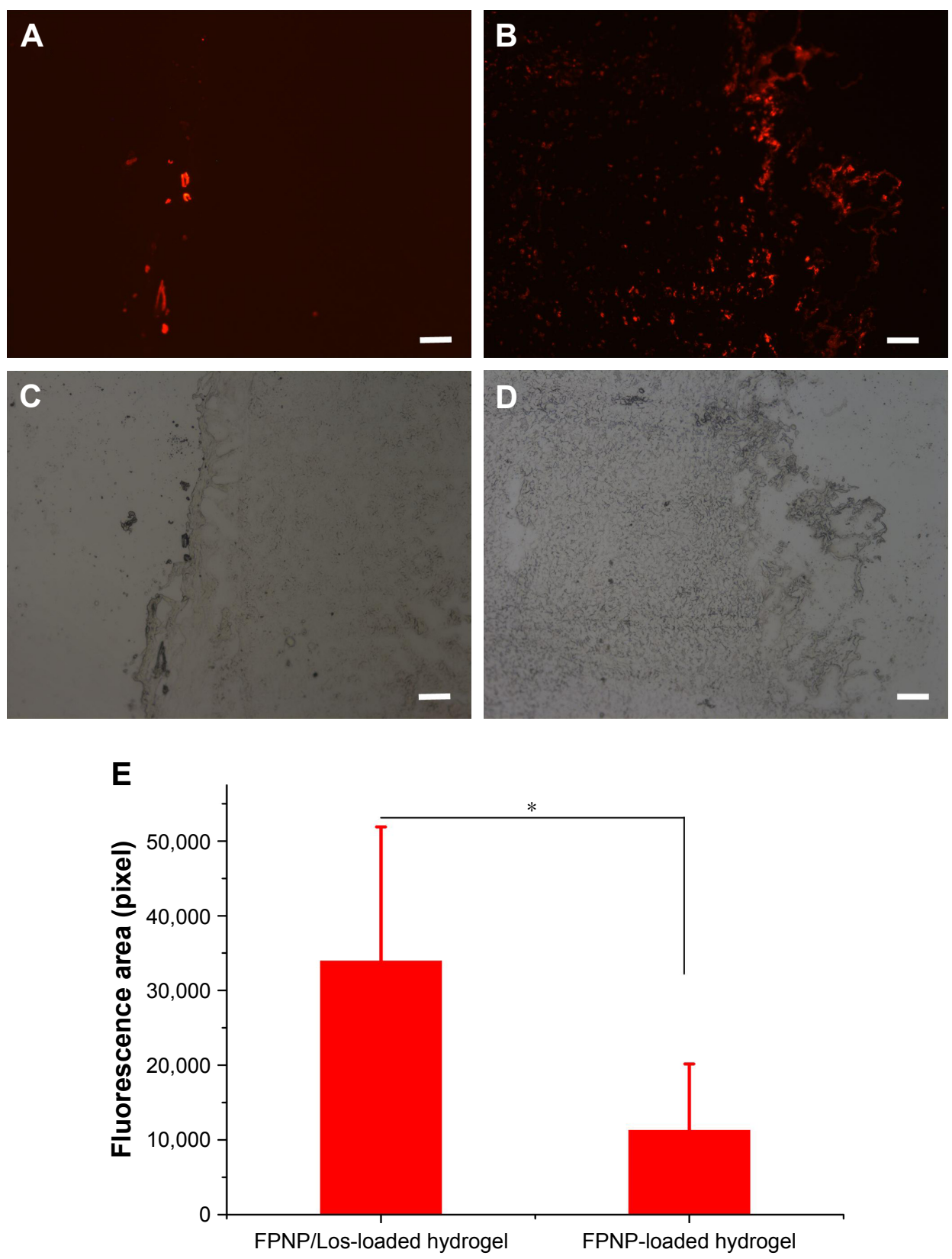

Figure 5 Fluorescent images of intratumoral distribution after peritumoral implantation of (A) FPNP-loaded hydrogel and (B) FPNP/Los-loaded hydrogel for 2 weeks. (C) Bright-field image of (A). (D) Bright-field image of (B). (E) Quantitative analysis of nanoparticle distribution with Image ( $\mathrm{n}=9$, mean \pm SD).

Notes: Scale bar represents $100 \mu \mathrm{m}$; magnification 100×. $* P<0.05$.

Abbreviations: FPNPs, fluorescent polystyrene nanoparticles; Los, losartan.

The distribution of collagen in tumor tissues was determined by collagen-specific Masson's trichrome staining, wherein the collagen content in tumor sections appears blue in color. The distinct collagen fiber network in the FPNPloaded hydrogel group was well organized and showed a high density of collagen (Figure 6A and C). This dense network may greatly restrict the distribution of nanoparticles in tumors. ${ }^{16}$ When treated with Los, in sharp contrast, the collagen bundles in the 4T1 tumors were fewer and less organized (Figure 6B and D). Compared to FPNP-loaded hydrogel, Los treatment showed a 3.56-fold reduction in the collagen expression (Figure 6E). This diminished expression and disturbed alignment of collagen may improve the permeability of tumors, thereby enhancing the distribution and penetration of nanoparticles. ${ }^{17,24}$

\section{Preparation and characterization of Doxil/Los-loaded hydrogel}

Because the enhanced distribution and penetration of nanoparticles had been confirmed as described earlier, the 

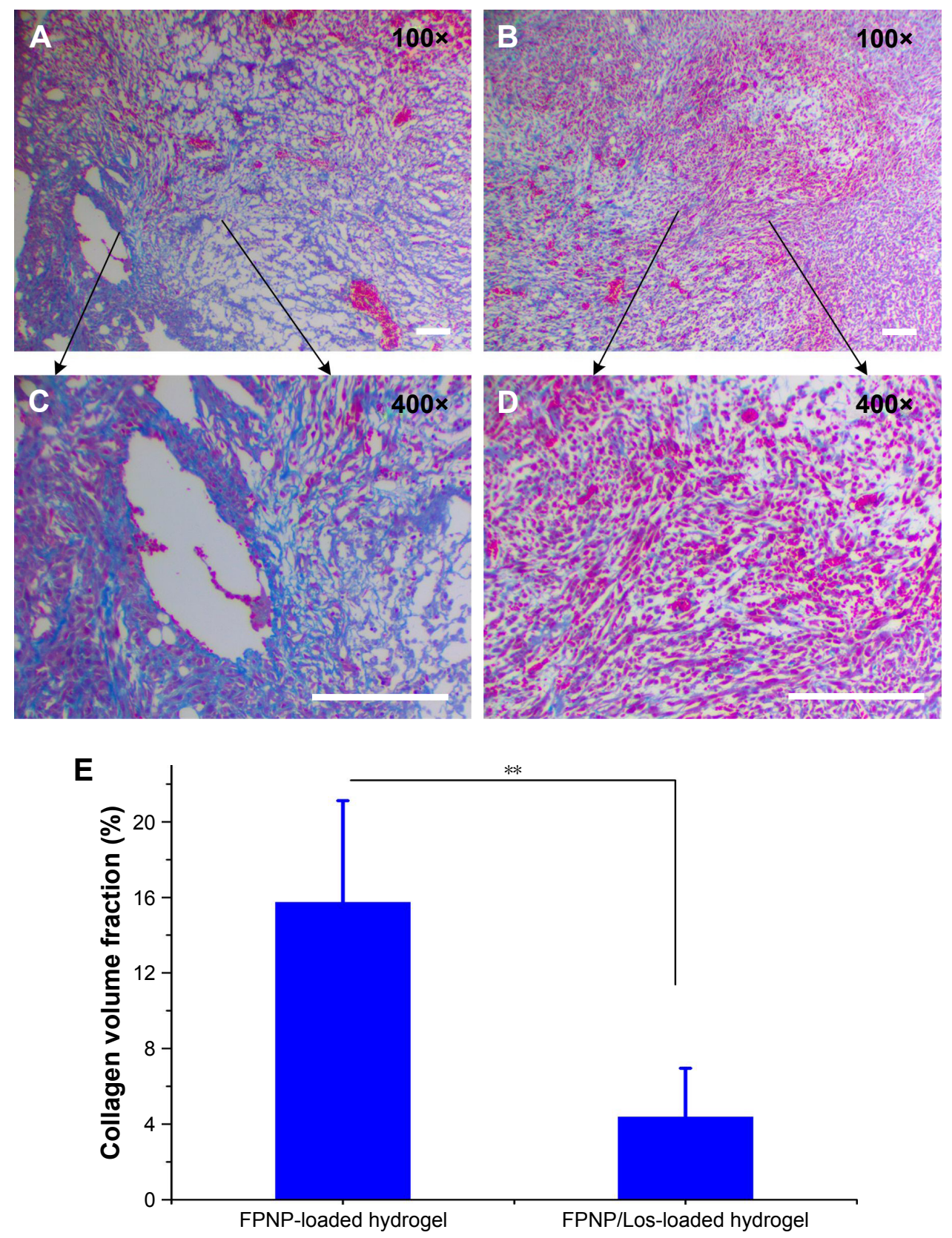

Figure 6 Representative images of Masson's trichrome staining of 4TI tumor from (A and $\mathbf{C})$ the FPNP-loaded hydrogel group and (B and D) FPNP/Los-loaded hydrogel group after treatment. (E) Quantification of Masson trichrome-stained collagen expression with ImageJ ( $n=6$, mean \pm SD).

Notes: The histopathological analysis was carried out at 100× and 400× magnification. Blue staining indicates collagen expression. Scale bar represents $100 \mu \mathrm{m}$. $* * P<0.01$. Abbreviations: FPNPs, fluorescent polystyrene nanoparticles; Los, losartan.

possibility of applying nanoparticles/Los-loaded hydrogels to cancer treatment by peritumoral implantation was then evaluated. Herein, Doxil was chosen to replace FPNPs to be encapsulated in hydrogel, because Doxil has a mean particle size of $\sim 100 \mathrm{~nm}$ - nearly equal to that of FPNPs. In addition, the improved distribution and penetration behavior observed for FPNPs in tumors should also be similar for Doxil. Moreover, Doxil has been approved for use as a chemotherapeutic agent for cancer patients. Thus, the antitumor effects of this nanoparticle formulation of DOX were well established, making it suitable to directly study the antitumor effects of Doxil-loaded hydrogels in the presence or absence of Los.

Doxil/Los-loaded hydrogels were formed by a thiolmaleimide reaction of 4-arm PEG-Mal with the crosslinker HS-MMP-SH. Prior to the reaction, a hydrogel precursor solution was made by evenly mixing 4-arm PEG-Mal with Doxil and Los (Figure 7A). After the addition of HS-MMP-SH and subsequent vortexing, a non-liquid and transparent brownish 

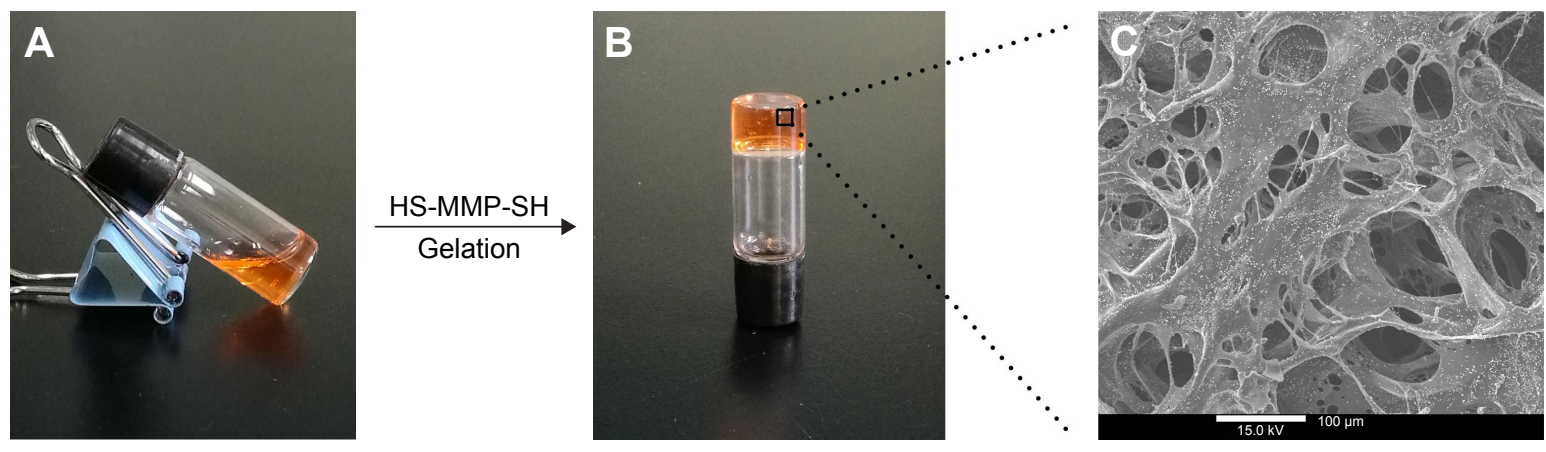

Figure 7 (A) Photograph of the hydrogel precursor solution containing 4-arm PEG-Mal, Doxil, and Los. (B) Photograph of the Doxil/Los-loaded hydrogel. (C) SEM image of the Doxil/Los-loaded hydrogel.

Notes: HS-MMP-SH, matrix metalloproteinases cleavable peptide. Magnification 200x.

Abbreviations: Doxil, liposomal doxorubicin; Los, losartan; PEG-Mal, polyethylene glycol maleimide; SEM, scanning electron microscopy.

red hydrogel was generated (Figure 7B). The porous network of hydrogel was clearly observed under SEM (Figure 7C). The interconnected pore diameter was approximately $44.34 \pm 19.87 \mu \mathrm{m}$ - much larger than the particle size of Doxil. Therefore, the porous network of hydrogel was suitable for the encapsulation of Doxil and Los. The red appearance of the hydrogel visually demonstrated the presence of Doxil.
Following the preparation of the Doxil/Los-loaded hydrogel, we examined the structural integrity of the Doxil incorporated in the hydrogel by comparing the size and zeta potential of the Doxil released from hydrogel with those of unincorporated Doxil (Figure 8A and B). ${ }^{31}$ Dynamic light scattering measurements of Doxil from the hydrogel in the absence of collagenase showed a size distribution of $79.09 \pm 0.79 \mathrm{~nm}$ (polydispersity
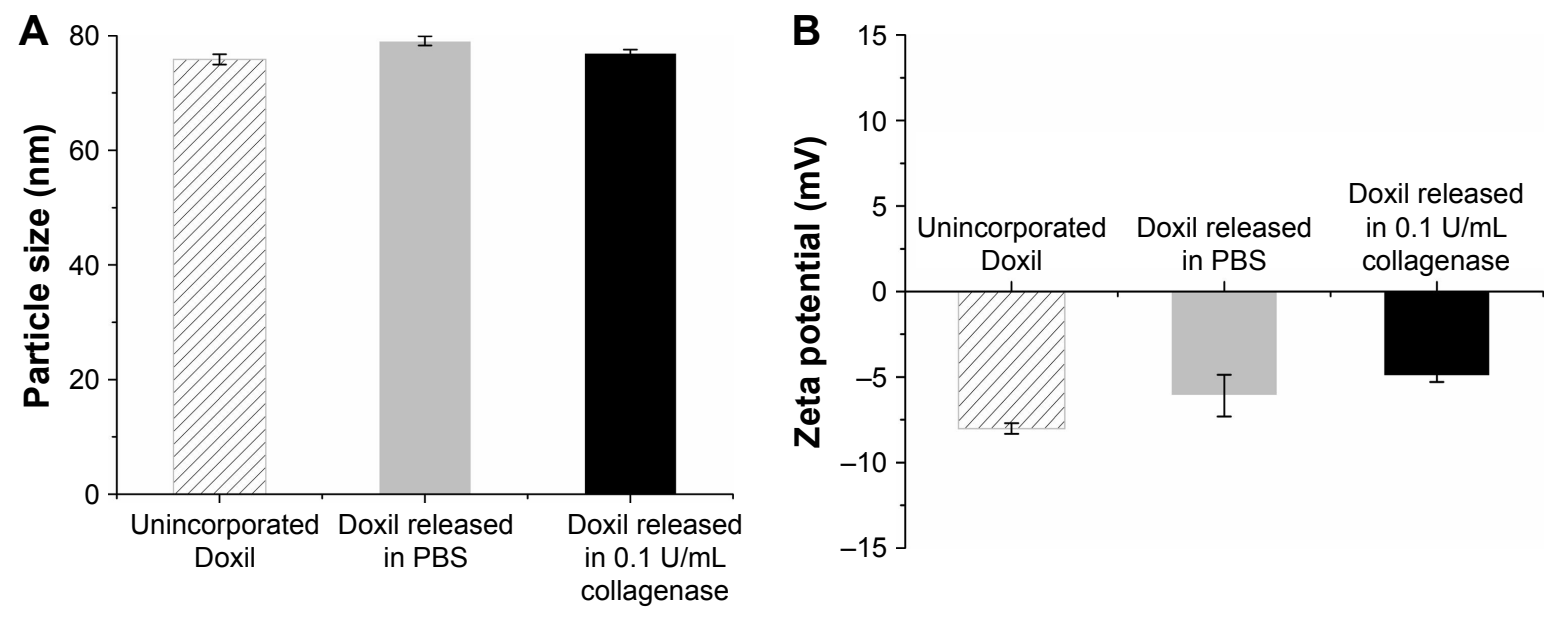

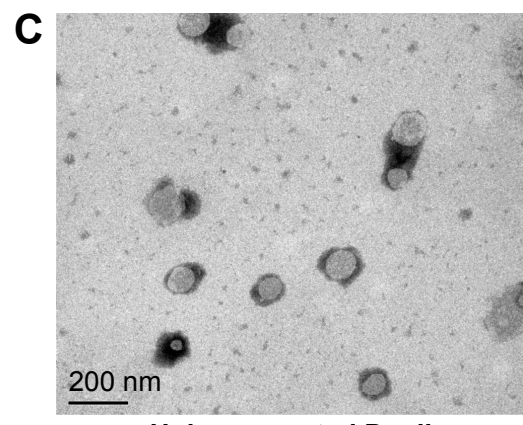

Unincorporated Doxil

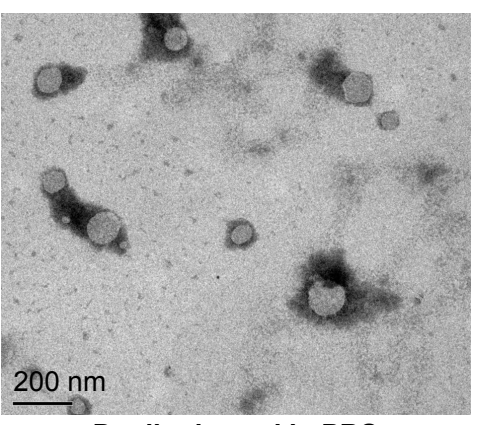

Doxil released in PBS

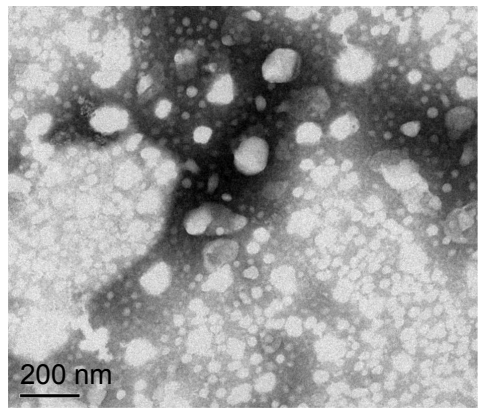

Doxil released in $0.1 \mathrm{U} / \mathrm{mL}$ collagenase

Figure 8 Particle size (A), surface zeta potential (B), and TEM images (C) of unincorporated Doxil and Doxil released from hydrogels in PBS medium with or without $0.1 \mathrm{U} / \mathrm{mL}$ collagenase.

Notes: Unincorporated Doxil refers to the Doxil prior to hydrogel incorporation. Particle size and zeta potential are presented as mean \pm SD ( $n=3$ ). TEM magnification $37,000 \times$. Abbreviations: Doxil, liposomal doxorubicin; TEM, transmission electron microscopy. 
index $=0.20 \pm 0.04$ ) and a zeta potential of $-6.09 \pm 1.22 \mathrm{mV}$. The measurements of the Doxil released in the presence of $0.1 \mathrm{U} / \mathrm{mL}$ collagenase showed a size distribution of $76.96 \pm 0.62 \mathrm{~nm}$ (polydispersity index $=0.09 \pm 0.01$ ) and a zeta potential of $-4.93 \pm 0.36 \mathrm{mV}$. These values matched those of the unincorporated Doxil, which showed a size distribution of $75.86 \pm 0.92 \mathrm{~nm}$ (polydispersity index $=0.05 \pm 0.02$ ) and a zeta potential of $-8.01 \pm 0.31 \mathrm{mV}$. These results suggest that the hydrogel developed in this study was able to preserve the integrity of Doxil during incorporation, and that intact Doxil was released from the hydrogel. This was further confirmed by TEM imaging (Figure $8 \mathrm{C}$ ). Nanoparticles with diameter $\sim 100 \mathrm{~nm}$, similar to the unincorporated Doxil, were dispersed in the release medium. Considering that there were no other kinds of nanoparticles in the hydrogel, the nanoparticles in the release medium were most likely to be Doxil.

\section{Cellular uptake of DOX from hydrogel}

The cellular uptake behavior of DOX from hydrogel was observed by the Cell Imaging System. A fluorescence marker, LysoTracker Green, was chosen to selectively stain endosomes and early lysosomes. Figure 9A shows the intracellular distribution of DOX in 4T1 cells after incubation with the Doxil-loaded and Doxil/Los-loaded hydrogels, respectively. In the Doxil-loaded hydrogel group, the overlap with red DOX signals and the green fluorescence of LysoTracker indicated that endocytosis was indeed involved in the uptake of DOX. The red fluorescence of DOX was scattered within the cytoplasm and the nuclear region, demonstrating that DOX was released from the hydrogel and internalized into cancer cells, and entered the nucleus where it could exert its cytotoxic effect. In the Doxil/Los-loaded hydrogel group, the intracellular distribution of DOX was very similar to that

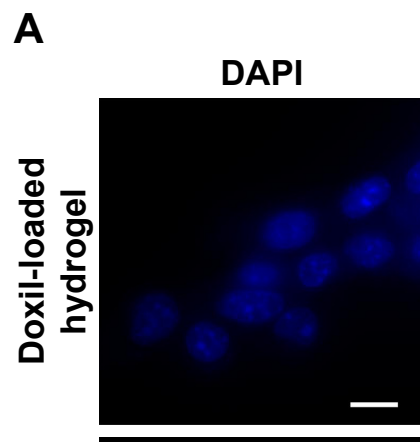

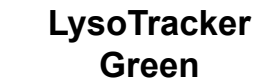
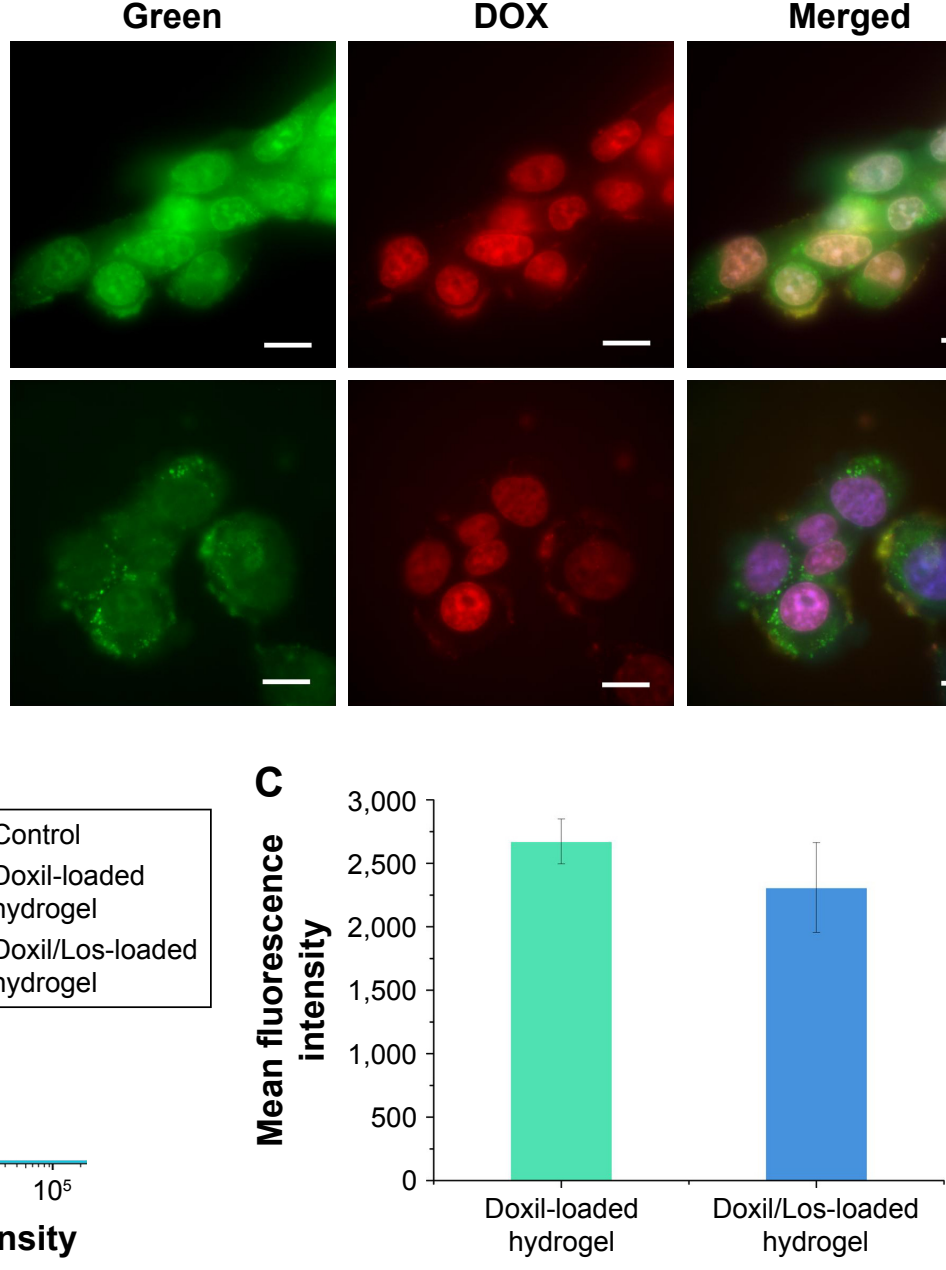

B

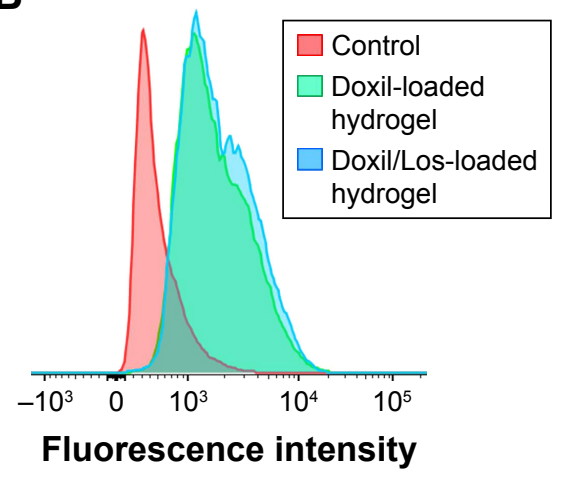

Figure 9 Cellular uptake analysis of DOX from Doxil-loaded hydrogel and Doxil/Los-loaded hydrogel.

Notes: (A) Fluorescence images showing the intracellular distribution of DOX. (B) Flow cytometric analysis of fluorescence intensity of DOX in cells. (C) Mean fluorescence intensity of DOX in cells analyzed by flow cytometry $(n=3$, mean \pm SD). Scale bar is $10 \mu \mathrm{m}$. The images were obtained under Cell Imaging System with $60 \times$ objective lens. There was no significant difference between the mean fluorescence intensities of the two groups shown in $(\mathbf{C}) ;(P>0.1)$.

Abbreviations: DAPI, 4',6-diamidino-2-phenylindole; DOX, doxorubicin; Doxil, liposomal doxorubicin; Los, losartan. 
of the Doxil-loaded hydrogel group, indicating that DOX could still be internalized into cancer cells in the presence of Los. To further investigate the influence of Los on cellular uptake, the fluorescence intensity of DOX in 4T1 cells was quantitatively assessed by flow cytometry. As shown in Figure 9B, the fluorescence in both Doxil-treated groups was obviously higher than that in the control group, demonstrating the release of DOX from the hydrogels and subsequent cellular uptake. There was no significant difference in fluorescence between the Doxil-loaded hydrogel group and Doxil/Los-loaded hydrogel group (Figure 9C), suggesting that Los did not affect the cellular uptake of DOX. Moreover, because collagenase could facilitate hydrogel degradation and drug release, the cellular uptake was studied in culture medium with $0.1 \mathrm{U} / \mathrm{mL}$ collagenase (Figure $\mathrm{S} 1$ ). As shown in Figure $\mathrm{S} 1$, this led us to the same conclusion as above. Taken together, all of these results verified the cellular uptake of DOX from hydrogels and the negligible influence of Los, which were crucial to our further studies of cytotoxicity and tumor growth inhibition.

According to previous reports, various classes of chemotherapeutics, including anthracyclines (eg, free DOX), are commonly taken up by cells through passive diffusion across cell membranes, whereas nanoparticle vectors penetrate into the cytosol via endocytosis. ${ }^{32-34}$ Herein, endocytosis was involved in the uptake of DOX, according to the results shown in Figure 9A, suggesting that the majority of DOX might be released from the hydrogel and taken up in the form of Doxil nanoparticles. This is reasonable, considering that intact Doxil was shown to be released from the hydrogel (see "Preparation and characterization of Doxil/Los-loaded hydrogel").

\section{In vitro cytotoxicity assay}

First, the in vitro cytotoxicity of hydrogels, with or without drugs, was qualitatively investigated by a live/dead assay, wherein live cells are stained green whereas dead cells are stained red. The results are shown in Figure 10A. After incubation with blank hydrogels for 24 hours in either the presence or absence of collagenase, most of the cells showed green fluorescence and grew well, demonstrating that the blank hydrogel did not have significant cytotoxicity. By contrast, after coculture with the Doxil-loaded and Doxil/Los-loaded hydrogel in the absence of collagenase, the number of dead cells with a red signal visibly increased, indicating that both of the Doxil-loaded hydrogels could induce tumor cell death and inhibit cell growth. After incubation with both Doxil-loaded hydrogels in the presence of collagenase, cells were observed to shrink, become round, and adhere poorly, indicating enhanced cytotoxicity.

Further, the cell viability was studied by a standard CCK-8 assay. As shown in Figure 10B, more than $80 \%$ of the cells remained viable after 48-hour coculture with the blank hydrogel, suggesting that the blank hydrogel had good biocompatibility and no cytotoxicity, in good agreement with the live/dead assay. Then, cell viability after treatment with Doxil-loaded and Doxil/Los-loaded hydrogels was determined (Figure 10B). In both cases, there was a timedependent cytotoxic effect. After 24-hour coculture in the absence of collagenase, both groups exhibited slightly lower cell viability than the blank hydrogel group, indicating an inhibitory effect against 4T1 tumor cells. By contrast, the cell viability was significantly lower than that of the blank hydrogel group after 48 -hour coculture $(P<0.05)$, indicating more effective inhibition. Similarly, in the presence of collagenase, the decrease in cell viability after 48-hour coculture $(P<0.01)$ was greater than that after 24 -hour coculture $(P<0.05)$. Considering that Doxil needs to be released from hydrogels before diffusing into the nucleus and causing cytotoxicity, the time-dependent cytotoxic effect is reasonable. Moreover, because collagenase facilitates the hydrogel degradation and Doxil release, the cell viability after treatment with both Doxil-loaded hydrogels in the presence or absence of collagenase was examined (Figure 10B). Compared with treatment without collagenase, the cell viability after treatment with collagenase was visibly lower, especially after 48 -hour coculture $(P<0.01)$. The enhanced cytotoxicity of both hydrogels in the presence of collagenase could be ascribed to the accelerated release and, consequently, higher concentration of DOX in the culture medium. Taken together, these results suggest that the introduction of Doxil nanoparticles and Los into PEG hydrogels did not affect the intrinsic chemotherapy effect of Doxil. Both the Doxil-loaded and Doxil/Los-loaded hydrogels had antitumor effects in vitro.

\section{Tumor growth inhibition in vivo}

The antitumor effect of the Doxil/Los-loaded hydrogel was evaluated on 4T1 xenograft-bearing mouse models. As shown in Figure 11B, the tumor growth speed after treatment with blank hydrogels was approximately equal to that of the control group, indicating that both blank hydrogel and surgical operation for implantation had negligible inhibitory effects on tumor growth. Doxil-loaded hydrogel treatment could slightly decrease the tumor growth speed compared with the control group, demonstrating that Doxil was 

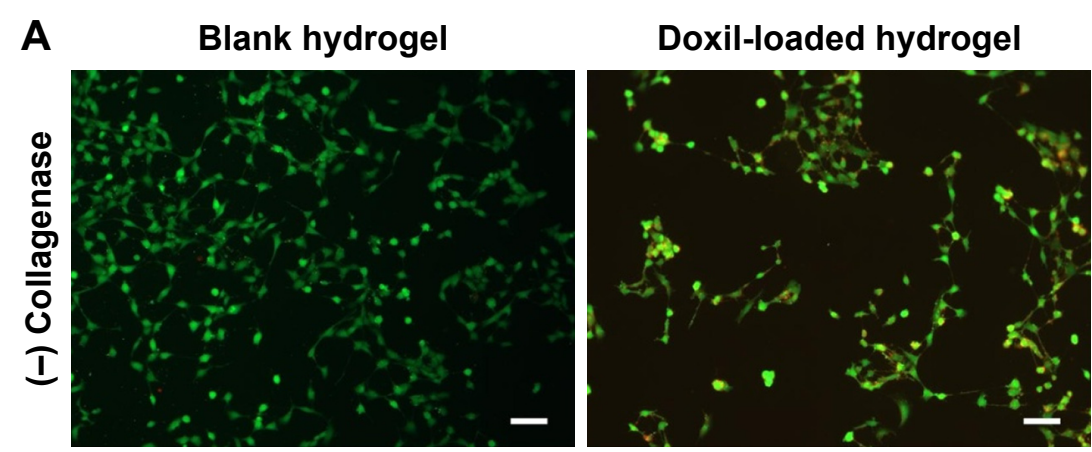

\section{Doxil/Los-loaded hydrogel}
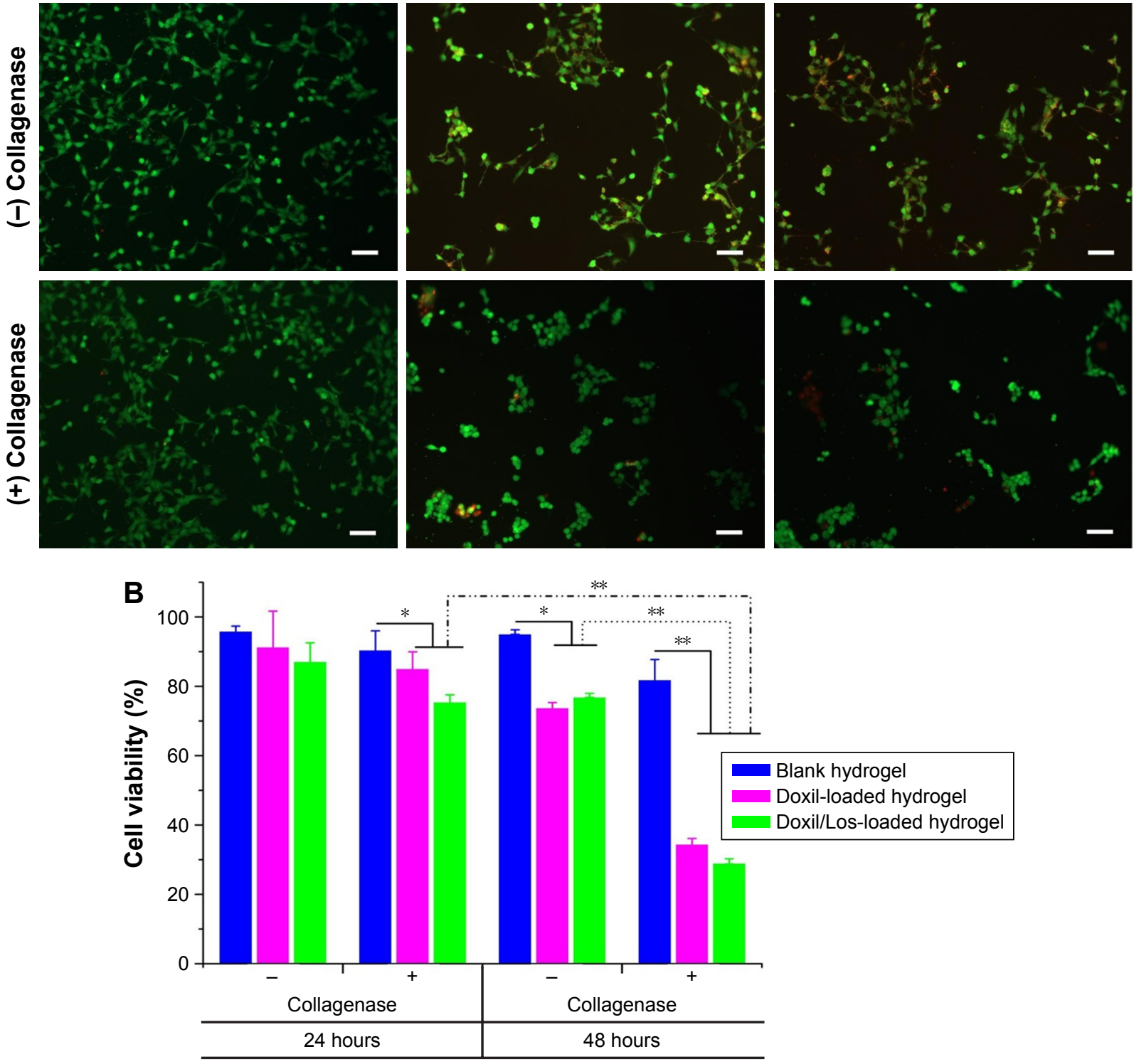

Figure 10 In vitro cytotoxicity of blank hydrogel and both Doxil-loaded hydrogels.

Notes: (A) Live/dead staining of 4TI cells after 24-hour incubation with different hydrogels in the absence $(-)$ or presence $(+)$ of collagenase. (B) Cell viability of 4TI cells after treatment with different hydrogels for 24 and 48 hours in the absence $(-)$ or presence $(+)$ of collagenase $(n=3$, mean \pm SD). For live/dead staining, green represents live cells and red represents dead cells. Scale bar is $50 \mu \mathrm{m}$; magnification $200 \times * P<0.05, * * P<0.01$.

Abbreviations: Doxil, liposomal doxorubicin; Los, losartan.

released from the hydrogel, delivered to the tumor site, and displayed an antitumor effect. However, the tumor growth was more rapid than that of the Doxil/Los-loaded hydrogel group (Figure 11B). The Doxil/Los-loaded hydrogel group showed the greatest inhibition. After treatment according to the study protocol, tumors were harvested, imaged, and weighed. The mice treated with blank hydrogels had large tumor volumes, whereas those treated with Doxil/ Los-loaded hydrogels displayed obviously reduced tumor size (Figure 11A). The inhibition rate of tumors, calculated from the tumor weights, is presented in Figure 11C. The
Doxil/Los-loaded hydrogel showed the highest inhibition rate of $59 \%$, whereas the Doxil-loaded hydrogel had a rate of only $22 \%$, suggesting that peritumoral implantation of the Doxil-loaded hydrogel in the presence of Los led to considerably better antitumor effects. Because Los treatment alone cannot reduce the growth rate of a tumor, and subsequent chemotherapy is necessary, ${ }^{16,19,25}$ the improved antitumor effect here can be attributed to Los decreasing the collagen content in the tumor stroma and consequently generating better penetration and distribution of nanoparticles (Doxil) in tumors. 
A

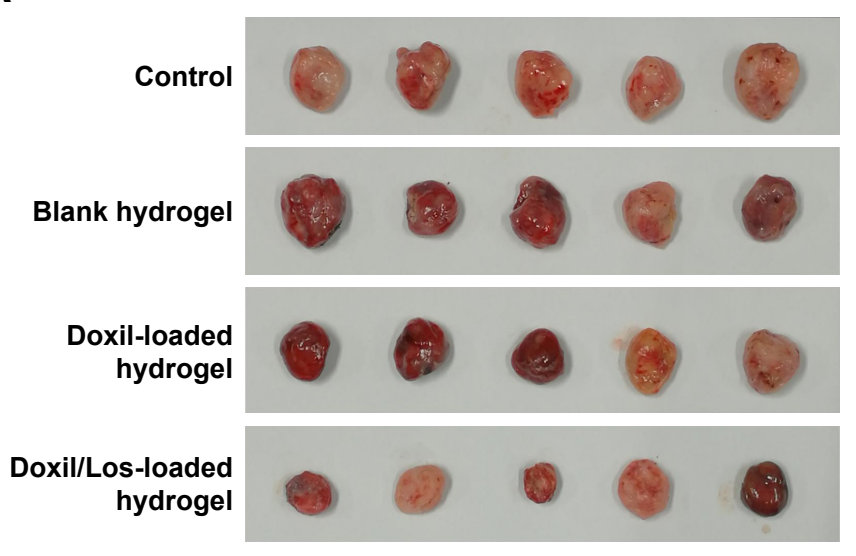

B

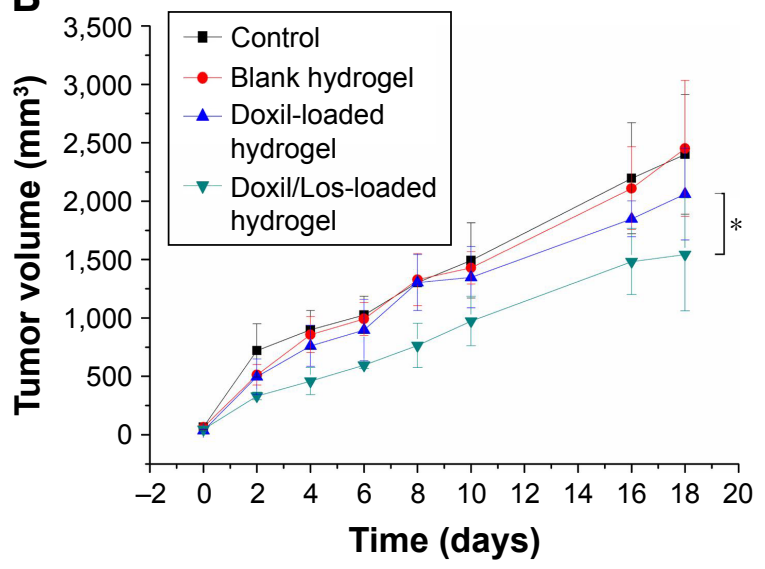

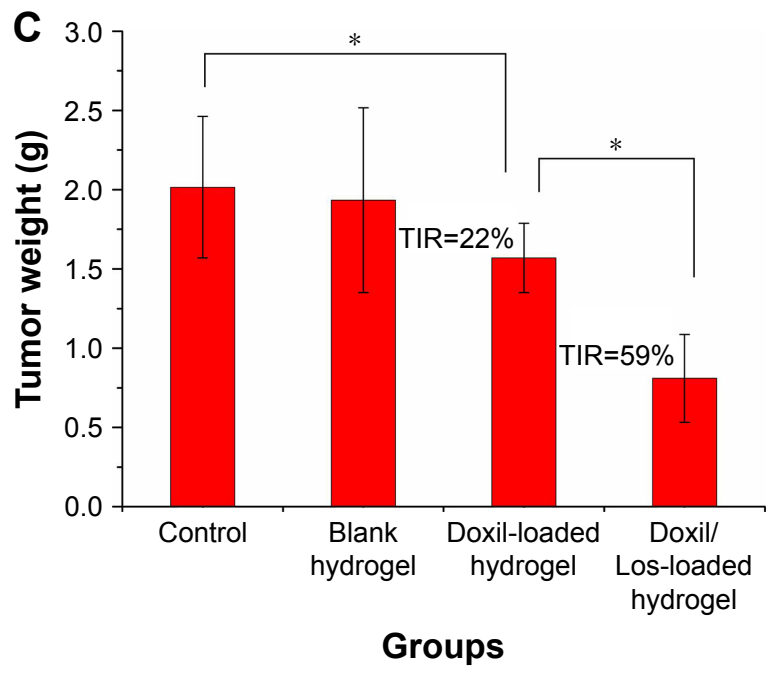

Figure II Therapeutic efficacy evaluation of different treatments on 4TI tumor growth in nude mice.

Notes: (A) Photographs of excised tumors from each treatment group after sacrifice at 18 days. (B) Primary tumor growth curves of mice receiving different treatments $(n=5$, mean $\pm S D)$. (C) Weight of excised tumors from each treatment group after sacrifice at 18 days ( $n=5$, mean \pm SD). Compared with the control group, the tumor weight after treatment with Doxil-loaded and Doxil/Los-loaded hydrogels was significantly reduced. The TIRs of the Doxil-loaded and Doxil/Los-loaded hydrogels were calculated as $22 \%$ and $59 \%$, respectively. $* P<0.05$.

Abbreviations: Doxil, liposomal doxorubicin; Los, losartan; TIR, tumor inhibitory rate.

\section{Discussion}

Nanoparticles offer promising approaches to the delivery of cancer therapeutics. Recently, implantation of nanoparticleloaded hydrogels in tumors has shown remarkable advantages in increasing nanoparticle concentration and exposure period in tumor sites. However, the intrinsic barriers generated by dense collagen networks seriously impaired the penetration of the released nanoparticles into tumors. This drawback significantly limited the therapeutic efficacy of nanoparticles. It is likely that, with deeper penetration into tumors and more nanoparticles internalized into tumor cells, better antitumor efficacy could be obtained. Therefore, it is important to overcome the intrinsic barriers to achieve more efficient drug delivery. Particle size is regarded as the most critical factor affecting penetration in the tumor interstitium. ${ }^{35,36}$ Generally, the permeability of nanoparticles decreases as particle size increases. Nanoparticles smaller than $50 \mathrm{~nm}$, such as gold nanoparticles and quantum dots, can penetrate poorly permeable tumors. ${ }^{29,35,37}$ However, most nanocarriers currently used for drug delivery have diameters of $\sim 100 \mathrm{~nm}$, including Doxil and Abraxane (diameters of 100 and $130 \mathrm{~nm}$, respectively), which are approved by the Food and Drug Administration for tumor treatment. Therefore, strategies for depleting intrinsic barriers and enhancing penetration of larger nanoparticles might have wide applicability. As a trial, we incorporated Los into nanoparticle-loaded hydrogels, expecting that the released Los would reduce the collagen level in tumors and pave the way for deeper distribution of nanoparticles. 
First, we constructed an implantable PEG hydrogel crosslinked by MMP-cleavable peptides. PEG has been widely used for drug delivery owing to its excellent biocompatibility. The MMP-cleavable peptide was selected because of the overexpressed MMPs in tumor sites. Thus, the employment of PEG and the MMP-cleavable peptide was expected to endow the hydrogel with remarkable biocompatibility and biodegradability. After implantation in tumor sites, the hydrogels would be gradually degraded and release their payloads. The release profile of the FPNPs (Figure 4) demonstrated the MMP-sensitive property of our hydrogel. Given that FPNPs with diameters of $\sim 100 \mathrm{~nm}$ could be released, it follows that the smaller Los molecules would also be released to exert their collagen-depleting effects.

To validate our proposed approach, the FPNP/Los-loaded hydrogel was implanted onto the surface of 4T1 tumor xenografts. As the tumor periphery is the most vascularized region, ${ }^{38}$ implanting the hydrogel at the peritumoral sites may favor the diffusion of nanoparticles and Los into the nearby blood vessels, directly delivering them alongside the blood flow into the tumor. ${ }^{11,13}$ Herein, the 4T1 xenograft tumor model was chosen owing to its high expression of collagen I. The main subtype of collagen, collagen I is highly strengthened, crosslinked, and remodeled during the progression of tumors. The collagen network in tumors is mainly constructed from collagen I. ${ }^{17,39}$ Los can dramatically deplete tumor collagen I, according to previous reports. ${ }^{16}$ Therefore, the 4T1 tumor model has been widely used to study the effects of collagen levels on nanoparticle penetration in tumors. ${ }^{19,25}$ Moreover, overexpression of MMP has been reported in 4T1 tumors, and is considered to contribute to their high metastatic ability. ${ }^{40,41}$ Thus, this tumor model was considered to be suitable for our present study. According to our results, after implantation of FPNP/Los-loaded hydrogel for 2 weeks, the intrinsic barrier of collagen was weakened (Figure 6) and the distribution of FPNPs in the tumor was markedly increased (Figure 5), directly demonstrating the validity of our approach.

On this basis, a Doxil/Los-loaded hydrogel was then developed for further antitumor studies. First, to determine whether Doxil was released from the hydrogel and Los inhibited the cytotoxicity of DOX, which were two crucial issues for further antitumor study, the cellular uptake and in vitro cytotoxicity were assessed. The cellular uptake and in vitro cytotoxicity results suggested that Doxil was released from the hydrogel, internalized into cells, and induced apoptosis of tumor cells. The in vivo antitumor study indicated that implantation treatment with the Doxil/Los-loaded hydrogel was more effective than treatment with the Doxil-loaded hydrogel in 4T1 tumor-bearing mice. These results were in good agreement with previous reports. For example, Godugu et al demonstrated that inhalational delivery of Los for 4 weeks to tumor-bearing animals could improve nanoparticle intratumoral distribution and anticancer effects in orthotopic and metastatic lung tumor models. ${ }^{24} \mathrm{Cun}$ et al showed that pretreatment with Los significantly decreased the collagen level and improved the penetration of DOX-loaded nanoparticles in 4T1 breast tumor. On co-administration of Los with DOX-loaded nanoparticles, the chemotherapeutic efficiency was significantly increased in comparison with treatment with DOX-loaded nanoparticles alone. ${ }^{17}$

It should be noted that, in our study, the peritumoral implantation of hydrogels was achieved by surgical operation. However, patient compliance with this method is unsatisfactory, owing to the suffering and high costs associated with surgery. Injectable hydrogels, including thermo-sensitive and self-healing hydrogels, might be promising candidates for better patient compliance. Therefore, it is worth developing an injectable hydrogel with good biocompatibility for encapsulating both nanotherapeutics and Los for local chemotherapy. Moreover, the inhibition of collagen synthesis and the resulting reduction of collagen levels in tumors do not occur immediately after Los treatment. This is a process that takes some time. In previous reports, pretreatment with Los was usually carried out for 2 weeks before intravenous injection of drug-loaded nanoparticles. The developed hydrogel, which could achieve sequential local delivery of Los and nanoparticles, will be also attractive.

\section{Conclusion}

In this study, we incorporated both Los and FPNPs into a PEG hydrogel to obtain an FPNP/Los-loaded hydrogel for peritumoral implantation. The hydrogel was crosslinked by MMP-cleavable peptides and, therefore, it could be degraded by MMPs overexpressed in tumor sites. After implantation, Los effectively depleted collagen networks and increased the penetration of FPNPs in 4T1 tumors in vivo. These results support the hypothesis that peritumoral implantation of nanoparticles/Los-loaded hydrogels improves the intratumoral penetration of nanoparticles. On this basis, a Doxil/ Los-loaded hydrogel was fabricated and its applicability for peritumoral chemotherapy against 4T1 tumors was evaluated. As expected, the chemotherapeutic efficiency of the Doxil/ Los-loaded hydrogel was significantly increased in comparison to treatment with a Doxil-loaded hydrogel alone. Taken together, these results suggest that co-delivery of Los and 
nanoparticles by an implantable hydrogel could improve the intratumoral penetration of nanoparticles and potentiate their synergistic anticancer effects. Therefore, it is reasonable to say that local chemotherapy based on a hydrogel containing both nanoparticles and Los could be a promising strategy, especially for poorly permeable tumors.

\section{Acknowledgment}

This work was supported by the National Natural Science Foundation of China (grant no 81402870), Natural Science Foundation of Jiangsu Province for Youth (grant no BK20140579), China Postdoctoral Science Foundation (grant no 2015M571696), Jiangsu Province Postdoctoral Science Foundation (grant no 1401145C), Natural Science Foundation of the Higher Education Institutions of Jiangsu Province (15KJB350001), Scientific Research Foundation of Jiangsu University (grant no 14JDG046), and Zhenjiang Science and Technology Innovation Project (grant no SH2017043).

\section{Disclosure}

The authors report no conflicts of interest in this work.

\section{References}

1. Ferrari M. Cancer nanotechnology: opportunities and challenges. Nat Rev Cancer. 2005;5(3):161-171.

2. Zeineldin R, Syoufjy J. Cancer Nanotechnology: Opportunities for Prevention, Diagnosis, and Therapy. Methods Mol Biol. 2017;1530:3-12.

3. Liu Y, Xu CF, Iqbal S, Yang XZ, Wang J. Responsive Nanocarriers as an Emerging Platform for Cascaded Delivery of Nucleic Acids to Cancer. Adv Drug Deliv Rev. 2017;115:98-114.

4. Petros RA, Desimone JM. Strategies in the design of nanoparticles for therapeutic applications. Nat Rev Drug Discov. 2010;9(8):615-627.

5. Albanese A, Tang PS, Chan WC. The effect of nanoparticle size, shape, and surface chemistry on biological systems. Annu Rev Biomed Eng. 2012;14:1-16.

6. Koo H, Huh MS, Sun IC, et al. In vivo targeted delivery of nanoparticles for theranosis. Acc Chem Res. 2011;44(10):1018-1028.

7. De Souza R, Zahedi P, Allen CJ, Piquette-Miller M. Polymeric drug delivery systems for localized cancer chemotherapy. Drug Deliv. 2010; 17(6):365-375.

8. Wolinsky JB, Colson YL, Grinstaff MW. Local drug delivery strategies for cancer treatment: gels, nanoparticles, polymeric films, rods, and wafers. J Control Release. 2012;159(1):14-26.

9. Shen H, Li F, Wang D, et al. Chitosan-alginate BSA-gel-capsules for local chemotherapy against drug-resistant breast cancer. Drug Des Devel Ther. 2018;12:921-934.

10. Luo JW, Zhang T, Zhang Q, et al. A novel injectable phospholipid gel co-loaded with doxorubicin and bromotetrandrine for resistant breast cancer treatment by intratumoral injection. Colloids Surf B Biointerfaces. 2016;140:538-547.

11. Yang G, Wang J, Wang Y, Li L, Guo X, Zhou S. An implantable active-targeting micelle-in-nanofiber device for efficient and safe cancer therapy. ACS Nano. 2015;9(2):1161-1174.

12. Strong LE, Dahotre SN, West JL. Hydrogel-nanoparticle composites for optically modulated cancer therapeutic delivery. J Control Release. 2014;178:63-68.

13. Ding D, Zhu Z, Li R, et al. Nanospheres-incorporated implantable hydrogel as a trans-tissue drug delivery system. ACS Nano. 2011;5(4):2520-2534.
14. Netti PA, Berk DA, Swartz MA, Grodzinsky AJ, Jain RK. Role of extracellular matrix assembly in interstitial transport in solid tumors. Cancer Res. 2000;60(9):2497-2503.

15. Provenzano PP, Eliceiri KW, Campbell JM, Inman DR, White JG, Keely PJ. Collagen reorganization at the tumor-stromal interface facilitates local invasion. BMC Med. 2006;4(1):38.

16. Diop-Frimpong B, Chauhan VP, Krane S, Boucher Y, Jain RK. Losartan inhibits collagen I synthesis and improves the distribution and efficacy of nanotherapeutics in tumors. Proc Natl Acad Sci U S A. 2011;108(7):2909-2914.

17. Cun X, Ruan S, Chen J, et al. A dual strategy to improve the penetration and treatment of breast cancer by combining shrinking nanoparticles with collagen depletion by losartan. Acta Biomater. 2016;31: 186-196.

18. Barua S, Mitragotri S. Challenges associated with Penetration of Nanoparticles across Cell and Tissue Barriers: A Review of Current Status and Future Prospects. Nano Today. 2014;9(2):223-243.

19. Hu C, Liu X, Ran W, et al. Regulating cancer associated fibroblasts with losartan-loaded injectable peptide hydrogel to potentiate chemotherapy in inhibiting growth and lung metastasis of triple negative breast cancer. Biomaterials. 2017;144:60-72.

20. McKee TD, Grandi P, Mok W, et al. Degradation of fibrillar collagen in a human melanoma xenograft improves the efficacy of an oncolytic herpes simplex virus vector. Cancer Res. 2006;66(5):2509-2513.

21. Eikenes L, Bruland ØS, Brekken C, Davies Cde L. Collagenase increases the transcapillary pressure gradient and improves the uptake and distribution of monoclonal antibodies in human osteosarcoma xenografts. Cancer Res. 2004;64(14):4768-4773.

22. Liu J, Liao S, Diop-Frimpong B, et al. TGF- $\beta$ blockade improves the distribution and efficacy of therapeutics in breast carcinoma by normalizing the tumor stroma. Proc Natl Acad Sci U S A. 2012;109(41): 16618-16623.

23. Chauhan VP, Martin JD, Liu H, et al. Angiotensin inhibition enhances drug delivery and potentiates chemotherapy by decompressing tumour blood vessels. Nat Commun. 2013;4:2516.

24. Godugu C, Patel AR, Doddapaneni R, Marepally S, Jackson T, Singh M. Inhalation delivery of Telmisartan enhances intratumoral distribution of nanoparticles in lung cancer models. $J$ Control Release. 2013;172(1): 86-95.

25. Zhang L, Wang Y, Yang Y, et al. High Tumor Penetration of Paclitaxel Loaded $\mathrm{pH}$ Sensitive Cleavable Liposomes by Depletion of Tumor Collagen I in Breast Cancer. ACS Appl Mater Interfaces. 2015;7(18): 9691-9701.

26. Cam C, Segura T. Chemical sintering generates uniform porous hyaluronic acid hydrogels. Acta Biomater. 2014;10(1):205-213.

27. Kim I, Choi JS, Lee S, et al. In situ facile-forming PEG cross-linked albumin hydrogels loaded with an apoptotic TRAIL protein. $J$ Control Release. 2015;214:30-39.

28. Zhu L, Wang T, Perche F, Taigind A, Torchilin VP. Enhanced anticancer activity of nanopreparation containing an MMP2-sensitive PEG-drug conjugate and cell-penetrating moiety. Proc Natl Acad Sci U S A. 2013; 110(42): 17047-17052.

29. Wong C, Stylianopoulos T, Cui J, et al. Multistage nanoparticle delivery system for deep penetration into tumor tissue. Proc Natl Acad Sci USA. 2011;108(6):2426-2431.

30. Zhu L, Kate P, Torchilin VP. Matrix metalloprotease 2-responsive multifunctional liposomal nanocarrier for enhanced tumor targeting. ACS Nano. 2012;6(4):3491-3498.

31. Gao W, Vecchio D, Li J, et al. Hydrogel containing nanoparticlestabilized liposomes for topical antimicrobial delivery. ACS Nano. 2014;8(3):2900-2907.

32. Man HB, Kim H, Kim HJ, et al. Synthesis of nanodiamond-daunorubicin conjugates to overcome multidrug chemoresistance in leukemia. Nanomedicine. 2014;10(2):359-369.

33. Feng C, Rui M, Shen H, et al. Tumor-specific delivery of doxorubicin through conjugation of $\mathrm{pH}$-responsive peptide for overcoming drug resistance in cancer. Int J Pharm. 2017;528(1-2):322-333. 
34. Rui M, Qu Y, Gao T, Ge Y, Feng C, Xu X. Simultaneous delivery of anti-miR21 with doxorubicin prodrug by mimetic lipoprotein nanoparticles for synergistic effect against drug resistance in cancer cells. Int J Nanomedicine. 2017;12:217-237.

35. Cabral H, Matsumoto Y, Mizuno K, et al. Accumulation of sub-100 nm polymeric micelles in poorly permeable tumours depends on size. Nat Nanotechnol. 2011;6(12):815-823.

36. Chauhan VP, Stylianopoulos T, Martin JD, et al. Normalization of tumour blood vessels improves the delivery of nanomedicines in a size-dependent manner. Nat Nanotechnol. 2012;7(6):383-388.

37. Huo S, Ma H, Huang K, et al. Superior penetration and retention behavior of $50 \mathrm{~nm}$ gold nanoparticles in tumors. Cancer Res. 2013; 73(1):319-330.
38. Campbell RB. Tumor physiology and delivery of nanopharmaceuticals. Anticancer Agents Med Chem. 2006;6(6):503-512.

39. Fang M, Yuan J, Peng C, Li Y. Collagen as a double-edged sword in tumor progression. Tumour Biol. 2014;35(4):2871-2882.

40. Lv Y, Zhao X, Zhu L, et al. Targeting intracellular MMPs efficiently inhibits tumor metastasis and angiogenesis. Theranostics. 2018;8(10): 2830-2845.

41. Zhou R, Xu L, Ye M, Liao M, Du H, Chen H. Formononetin inhibits migration and invasion of MDA-MB-231 and 4T1 breast cancer cells by suppressing MMP-2 and MMP-9 through PI3K/AKT signaling pathways. Horm Metab Res. 2014;46(11):753-760. 


\section{Supplementary material}

A
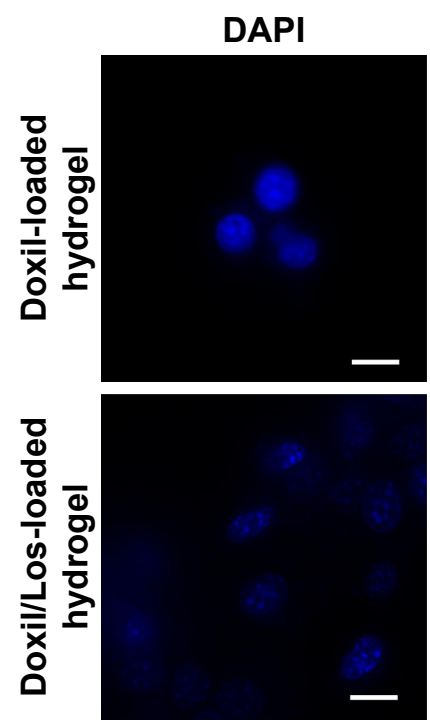

B

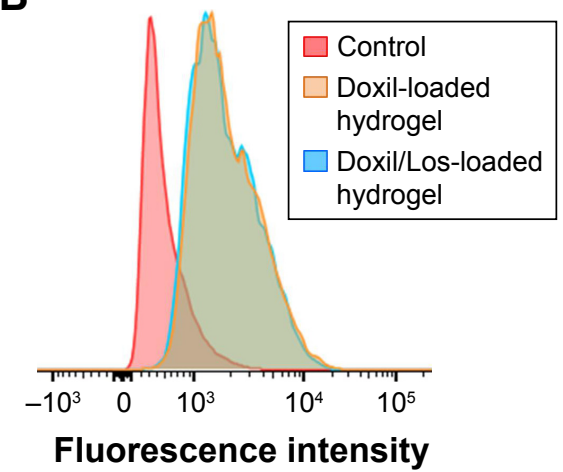

LysoTracker
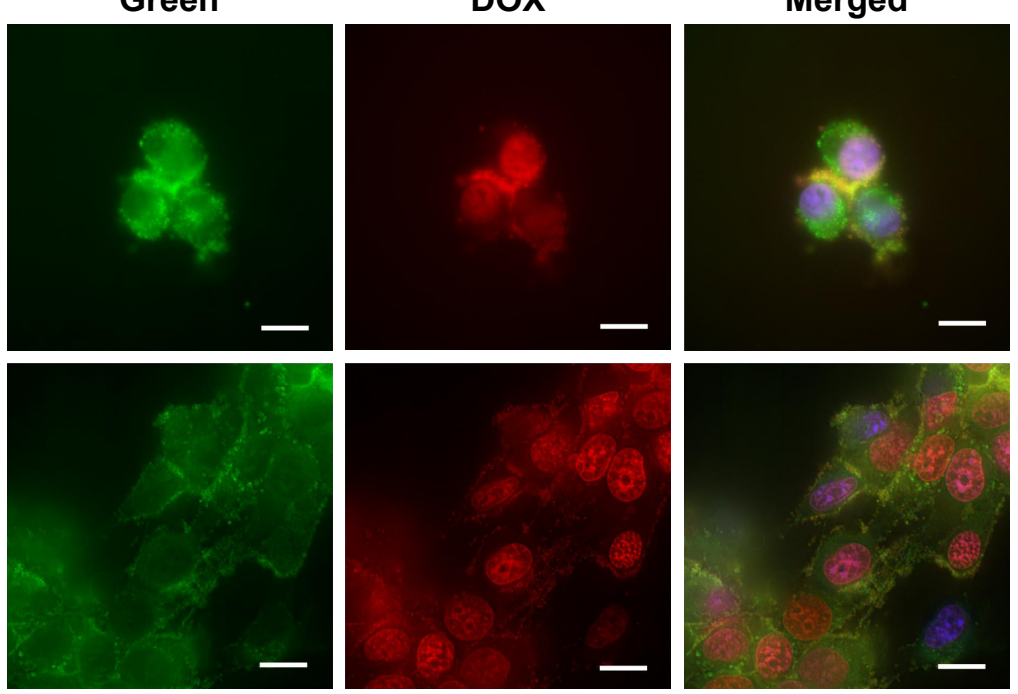

C

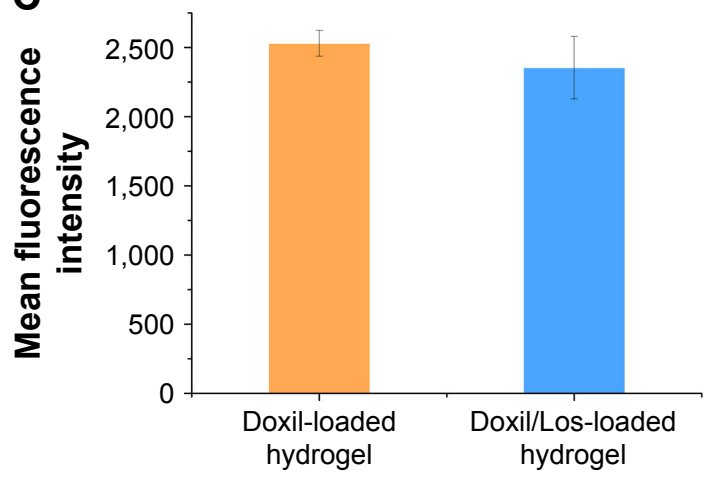

Figure SI Cellular uptake analysis of DOX from Doxil-loaded hydrogel and Doxil/Los-loaded hydrogel.

Notes: (A) Fluorescence images showing the intracellular distribution of DOX. (B) Flow cytometric analysis of fluorescence intensity of DOX in cells. (C) Mean fluorescence intensity of DOX in cells analyzed by flow cytometry $(n=3$, mean \pm SD). Scale bar is $10 \mu \mathrm{m}$. The images were obtained under Cell Imaging System with $60 \times$ objective lens. There was no significant difference between the mean fluorescence intensities of the two groups shown in (C). P $>0.1$.

Abbreviations: DAPI, 4',6-diamidino-2-phenylindole; DOX, doxorubicin; Doxil, liposomal doxorubicin; Los, losartan.

\section{Publish your work in this journal}

The International Journal of Nanomedicine is an international, peerreviewed journal focusing on the application of nanotechnology in diagnostics, therapeutics, and drug delivery systems throughout the biomedical field. This journal is indexed on PubMed Central, MedLine, CAS, SciSearch ${ }^{\circledR}$, Current Contents ${ }^{\circledR} /$ Clinical Medicine,
Journal Citation Reports/Science Edition, EMBase, Scopus and the Elsevier Bibliographic databases. The manuscript management system is completely online and includes a very quick and fair peer-review system, which is all easy to use. Visit http://www.dovepress.com/ testimonials.php to read real quotes from published authors. 